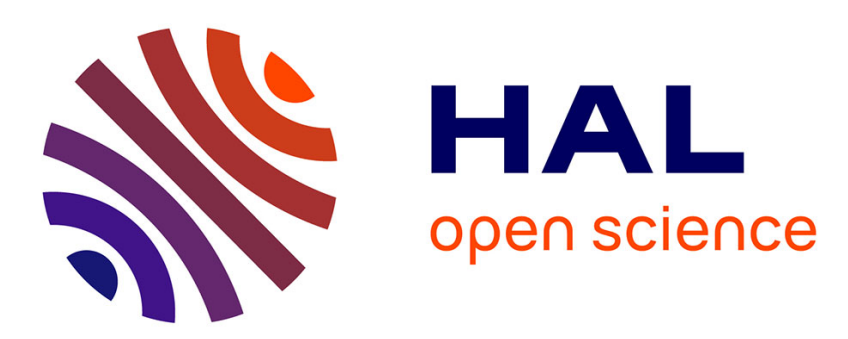

\title{
Dispersive smoothing for the Euler-Korteweg model
}

Corentin Audiard

\section{To cite this version:}

Corentin Audiard. Dispersive smoothing for the Euler-Korteweg model. SIAM Journal on Mathematical Analysis, 2012, 44 (4), pp.3018-3040. 10.1137/11083174X . hal-00786513

\section{HAL Id: hal-00786513 \\ https://hal.science/hal-00786513}

Submitted on 8 Feb 2013

HAL is a multi-disciplinary open access archive for the deposit and dissemination of scientific research documents, whether they are published or not. The documents may come from teaching and research institutions in France or abroad, or from public or private research centers.
L'archive ouverte pluridisciplinaire HAL, est destinée au dépôt et à la diffusion de documents scientifiques de niveau recherche, publiés ou non, émanant des établissements d'enseignement et de recherche français ou étrangers, des laboratoires publics ou privés. 


\title{
DISPERSIVE SMOOTHING FOR THE EULER-KORTEWEG MODEL*
}

\author{
CORENTIN AUDIARD ${ }^{\dagger}$
}

\begin{abstract}
The Euler-Korteweg system consists of a quasi-linear, dispersive perturbation of the Euler equations. The Cauchy problem has been studied in any dimension $d \geq 1$ by Benzoni, Danchin, and Descombes, who obtained local well-posedness results when the velocity is in $H^{s}$ for $s>d / 2+1$. They noticed that one may expect to find some smoothing effect due to the dispersive effects, but there was no proof so far. Our aim here is to give such results in any dimension under their local existence assumptions. In the simpler case of dimension 1 we obtain unconditionnal Kato smoothing (local smoothing of $1 / 2$ derivative). In higher dimensions a few additional hypotheses must be made to get smoothing and we briefly discuss the pertinence of these restrictions.
\end{abstract}

Key words. dispersive equations, Euler-Korteweg system, local smoothing, paradifferential calculus

AMS subject classifications. 35B65, 35Q30, 35Q55, 35S50, 76D45

DOI. $10.1137 / 11083174 \mathrm{X}$

1. Introduction. Dispersive smoothing is by now a rather classical topic. Its first observation originates with the seminal work of Kato on the Korteweg-de Vries equation [Kat83] (and almost at the same time Kruzhkov and Faminskiı [KF83]). There have since been various generalizations and refinements of these results for the Korteweg-de Vries equation [KPV91], as well as for very general dispersive equations [CS88]. Seemingly, the first result of dispersive smoothing for the Schrödinger equation with fully variable coefficients was obtained by Doi [Doi96] (different properties, of microlocal nature, were also obtained by Craig, Kappeler, and Strauss [CKS95]), who used geometric assumptions such as nontrapping of bicharacteristics and flatness of the coefficients at infinity that proved to be (in some sense) sharp. More recently, Doi's strategy of proof was successfully generalized by Kenig, Ponce, and Vega for the quasi-linear Schrödinger equation [KPV04] and Alazard, Burq, and Zuilly [ABZ] for (a convenient reformulation of) the one-dimensional water waves equations.

We treat here the Euler-Korteweg equations for capillary fluids, which read

$$
\left\{\begin{array}{l}
\partial_{t} \rho+\operatorname{div}(\rho u)=0 \\
\partial_{t} u+(u \cdot \nabla) u+\nabla g_{0}(\rho)=\nabla\left(K(\rho) \Delta \rho+\frac{1}{2} K^{\prime}(\rho)|\nabla \rho|^{2}\right)
\end{array}\right.
$$

where the right-hand side of the second equation modelizes capillarity forces. It is a dispersive perturbation of the classical Euler equations. Special solutions, namely, traveling profiles of the form $u(x \cdot \mathbf{n}-c t)$, have been known for a long time; see, for example, [BGDDJ07] for a review on the topic of their stability.

The analysis of the Cauchy problem for (EK) with general $K$ was initiated by Benzoni-Garage, Danchin, and Descombes in [BGDD06] in dimension 1; they later obtained in [BGDD07] the local well-posedness in any dimension $d$ for $\left.(\nabla \rho, u)\right|_{t=0} \in$

*Received by the editors April 21, 2011; accepted for publication (in revised form) June 14, 2012; published electronically August 16, 2012. Part of this work was done at the Basque Center for Applied Mathematics during the author's postdoc work in the frame of the NUMERIWAVES research project, ERC grant FP7-246775.

http://www.siam.org/journals/sima/44-4/83174.html

${ }^{\dagger}$ UPMC Univ Paris 6, UMR 7598, Laboratoire Jacques-Louis Lions, F-75005, Paris, France, and CNRS, UMR 7598, Laboratoire Jacques-Louis Lions, F-75005, Paris, France (corentin. audiard@ijil.math.upmc.org).

3018 
$H^{s}, s>d / 2+1$. The dispersive nature of this system is clarified by introducing the new unknown $\zeta=R(\rho)$, where $R$ is a primitive of the application $\rho \rightarrow \sqrt{K(\rho) / \rho}$. The system satisfied by $\zeta, u$ is then artificially supplemented by an equation on the unknown $w:=\partial_{x} \zeta$ obtained by differentiating in $x$ the transport equation on $\zeta$.

Consider first the simpler case of dimension 1 ; the equations on $(\rho, u)$ are

$$
\left\{\begin{array}{l}
\partial_{t} \rho+\partial_{x}(\rho u)=0, \\
\partial_{t} u+\left(u \cdot \partial_{x}\right) u+\partial_{x} g_{0}(\rho)=\partial_{x}\left(K(\rho) \partial_{x}^{2} \rho+\frac{1}{2} K^{\prime}(\rho)\left|\partial_{x} \rho\right|^{2}\right),
\end{array}\right.
$$

and the extended system reads

$$
\left\{\begin{array}{l}
\partial_{t} \zeta+u \partial_{x} \zeta+a(\zeta) \partial_{x} u=0 \\
\partial_{t} u+u \partial_{x} u-w \partial_{x} w-\partial_{x}\left(a(\zeta) \partial_{x} w\right)=-g^{\prime}(\zeta) w \\
\partial_{t} w+u \partial_{x} w+w \partial_{x} u+\partial_{x}\left(a(\zeta) \partial_{x} u\right)=0
\end{array}\right.
$$

where $g^{\prime}=R^{-1} / a \cdot g_{0}^{\prime} \circ R^{-1}, a(\zeta)=\sqrt{R^{-1}(\zeta) K\left(R^{-1}(\zeta)\right)}$.

One may identify the last two equations in (EEK) as the real and imaginary parts of a quasi-linear Schrödinger equation on $z=u+i w$ :

$$
\partial_{t} z+z \partial_{x} z+i \partial_{x}\left(a(\zeta) \partial_{x} z\right)=-g^{\prime}(\zeta) \operatorname{Re}(z) .
$$

The term $z \partial_{x} z$ will have to be treated with some care. Indeed, if we write (SB) as

$$
\partial_{t} z+u \partial_{x} z+i w \partial_{x} z+i \partial_{x}\left(a \partial_{x} z\right)=-g^{\prime}(\zeta) \operatorname{Re}(z),
$$

we see that (at least formally by Fourier modes analysis) $i w \partial_{x} z$ is a source of spectral instability. More precisely, if $w$ is independent of $t$, a criterion due to Mizohata [Miz81] requires that $\int_{0}^{X} w(x) d x$ remain uniformly bounded in $X$ for an $L^{2}$ estimate to stand. It is noticeable that this formal criterion is often satisfied here since $w$ is the derivative of $\zeta(\rho)$; thus it is bounded if $\rho$ is bounded. (This is required for the local existence theorem in [BGDD06].)

In a higher dimension, a different, but crucial, feature appears for the reformulated system

$$
\left\{\begin{array}{l}
\partial_{t} \zeta+u \cdot \nabla \zeta+a(\zeta) \operatorname{div} u=0 \\
\partial_{t} z+(u \cdot \nabla) z+i(\nabla z) \cdot w+i \nabla(a \operatorname{div} z)=-g^{\prime}(\zeta) \operatorname{Re}(z)
\end{array}\right.
$$

The second equation is no longer a quasi-linear Schrödinger equation but a degenerate quasi-linear Schrödinger equation. This fact prevents smoothing for general initial data and some (technical) cancellations of the one-dimensional case are not true anymore. Another important but more standard feature is the appearance of geometric assumptions, such as nontrapping of the bicharacteristics and some flatness at infinity of the symbol $\xi a(x) \xi^{t}$.

Smoothing properties of the Euler equations are interesting on a mathematical level because the degeneracy of the equations studied here raises new technical difficulties. On a more physical level it is still not known whether small perturbations of traveling waves solutions of (EK) exist globally or if they may blow up, the blowup criterion of [BGDDJ05] involving derivatives of too high an order. The slight smoothing proved here may be a further step to close the a priori estimates.

To prove our results, we use the gauge methods of [BGDD07] and follow closely the approach in [ABZ], which consists of reducing the quasi-linear problem to a linear one by means of paradifferential calculus. The ideas developped by Kenig, Ponce, and 
Vega [KPV04] for the quasi-linear Schrödinger equation are also important when the dimension is larger than 1.

The scheme of the proof can be described as follows. We have $z$ an $H^{s}$ solution, and the equations are reformulated as equations on some $Z_{s}$ which is exactly $s$ times less regular than $z$. A symbol $p$ is then constructed such that

$$
\frac{d}{d t}\left\langle T_{p} Z_{s}, Z_{s}\right\rangle \geq\left\|Z_{s} \varphi\right\|_{H^{1 / 2}}^{2}-\left\|Z_{s}\right\|_{L^{2}}^{2}
$$

with $\varphi$ having some decay at infinity. This estimate is true for smooth solutions and then implies the local $1 / 2$ derivative smoothing by density arguments.

Our paper is organized as follows:

- In section 2 we recall the essential results on paradifferential calculus that we use in the rest of the paper.

- In section 3 we treat the one-dimensional case by para-linearizing the equations.

- Section 4 extends to any dimension the results of section 3 under some supplementary assumptions when $\nabla \rho, u$ belong to Sobolev spaces.

- Since many physically pertinent solutions of the Euler-Korteweg system do not cancel at infinity, we extend our results to perturbations of such solutions in section 5. We briefly discuss the necessity of "flatness at infinity" and prove the smoothing effect for the linearized extended system near a traveling profile with weaker assumptions than in the general case.

- In the appendix we give for completeness the (relatively) standard arguments for the construction of paradifferential operators that are essential tools of the previous sections, and we give the proof of a weighted Gårding inequality.

2. Handtool in paradifferential calculus. Paradifferential calculus was introduced in the pioneering paper of Bony [Bon81]. It has since proved to be a very powerful tool for the analysis of nonlinear partial differential equations. For the results of this section we refer to the lecture notes of Métivier [Mét08]. ${ }^{1}$ Chapter 5 proves continuity and paralinearization properties of paradifferential operators; the formulas of symbolic calculus are proved in chapter 6 .

DeFINITION 2.1. Let $\chi(\eta, \xi)$ be a smooth nonnegative function. We say that it is an admissible truncature function when there exists $0<\varepsilon_{1}<\varepsilon_{2}<1$ such that

$$
\left\{\begin{array}{l}
\chi(\eta, \xi)=1,|\eta| \leq \varepsilon_{1}(1+|\xi|) \\
\chi(\eta, \xi)=0,|\eta| \geq \varepsilon_{2}(1+|\xi|)
\end{array}\right.
$$

and for any multi-indices $(\alpha, \beta)$ there exists a constant $C_{\alpha, \beta}>0$ such that $\left|\partial_{\eta}^{\alpha} \partial_{\xi}^{\beta} \chi\right| \leq$ $C(1+|\xi|)^{-|\alpha|-|\beta|}$. Following Example 5.1.5 of [Mét08], we will choose for $\chi$

$$
\chi(\eta, \xi)=\sum \varphi_{k}(\xi) \theta_{k-3}(\eta)
$$

where $\theta_{k}(\xi)=\theta\left(2^{-k} \xi\right), \varphi_{0}=\theta_{0}, \forall k \geq 1, \varphi_{k}=\theta_{k}-\theta_{k-1}$, and $\theta$ satisfies

$$
\theta \in C_{c}^{\infty}\left(\mathbb{R}^{d}\right), \theta=1 \text { for }|\xi| \leq 1.1, \theta=0 \text { for }|\xi| \geq 1.9 \text {. }
$$

For $r \geq 0, m \in \mathbb{R}$, the space of symbols $\Gamma_{r}^{m}$ is the set of functions $a(x, \xi): \mathbb{R}^{d} \times \mathbb{R}^{d} \rightarrow$ $\mathbb{C}, C^{\infty}$ in $\xi \neq 0, W^{r, \infty}$ in $x$ for any $\xi \neq 0$ such that for any multi-index $\alpha$ and any $|\xi| \geq 1 / 2,\left\|\partial_{\xi}^{\alpha} a(\cdot, \xi)\right\|_{W^{r, \infty}} \leq C_{\alpha}(1+|\xi|)^{m-|\alpha|}$.

\footnotetext{
${ }^{1}$ We use the chapter numbering of the pdf file available on the webpage of Métivier, http://www.math.u-bordeaux.fr/metivier/coursinE.html.
} 
Remark 2.2. In particular the Sobolev embeddings ensure that if $a(x) \in H^{s}\left(\mathbb{R}^{d}\right)$, $s>r+d / 2$, then $a \in \Gamma_{r}^{0}$.

Definition 2.3 (see [Mét08, Definition 5.1.14]). Let $\psi \in C^{\infty}\left(\mathbb{R}^{d} ; \mathbb{R}^{+}\right)$be such that $\forall|\eta| \leq 1, \psi(\eta)=0, \forall|\eta| \geq 2, \psi(\eta)=1$. For $a \in \Gamma_{r}^{m}$ we define the paradifferential operator $T_{a}$ by

$$
\widehat{T_{a} u}(\xi)=\frac{1}{(2 \pi)^{d}} \int_{\mathbb{R}^{d}} \chi(\xi-\eta, \eta) \psi(\eta) \widehat{a}(\xi-\eta, \eta) \widehat{u}(\eta) d \eta,
$$

where $\widehat{a}$ is the Fourier transform of a with respect to the variable $x$.

Equivalently, if $G_{\chi}$ is the inverse Fourier transform with respect to $\eta$ of $\chi(\eta, \xi)$, $T_{a}$ is the pseudodifferential operator of symbol $\int G_{\chi}(x-y, \xi) a(y, \xi) d y \psi(\xi) /(2 \pi)^{d}$. (This less explicit definition has the advantage of making sense also when $\widehat{a}$ is only a distribution.)

Remark 2.4. Our definition is slightly different from the one given in the beginning of chapter 5 in [Mét08]. The presence of the function $\psi$ is necessary to deal with symbols which are not smooth in $\xi$ near 0; however, it is only introduced in [Mét08] at the end of chapter 6 (section 6.4). This is a mostly harmless modification, but - as pointed out by the reviewer - it raises a few modifications in the paralinearization results that we describe hereafter.

Proposition 2.5 (Theorem 5.1.15 in [Mét08]). If $a \in \Gamma_{r}^{m}, r \geq 0$, then $T_{a}$ is continuous $H^{s+m} \rightarrow H^{s}$ for $s \in \mathbb{R}$, and

$$
\left\|T_{a}\right\|_{H^{s+m} \rightarrow H^{s}} \lesssim \sup _{|\xi| \geq 1 / 2,|\alpha| \leq d / 2+1}(1+|\xi|)^{|\alpha|-m}\left\|\partial_{\xi}^{\alpha} a(\cdot, \xi)\right\|_{L^{\infty}} .
$$

Proposition 2.6 (paralinearization formulas).

1. If $u, v \in H^{s} \times H^{r}, s, r>d / 2$, then

$$
u v=T_{u} v+T_{v} u+R(u, v)
$$

with $R(u, v) \in H^{s+r-d / 2},\|R(u, v)\|_{H^{s+r-d / 2}} \leq C\|u\|_{H^{s}}\|v\|_{H^{r}}$.

2. If $a$ and $u$ are bounded, $\nabla a \in H^{s-1}, u \in L^{2}, s \geq 1$, then

$$
\left\|a u-T_{a} u\right\|_{H^{s}} \lesssim\|\nabla a\|_{H^{s-1}}\|u\|_{L^{\infty}}+\|a\|_{L^{\infty}}\|u\|_{L^{2}} .
$$

3. If $a \in W^{r, \infty}, r$ integer $\geq 1, u \in L^{2}$, then

$$
\left\|a \partial_{x} u-T_{a} \partial_{x} u\right\|_{H^{r-1}} \lesssim\|a\|_{W^{r, \infty}}\|u\|_{L^{2}} .
$$

Proof. This is the only part of this section where the proofs of [Mét08] need modifications. We note $\widetilde{T_{a}}$ the paradifferential operator as defined in section 5 of [Mét08]; this is (2.1) without the $\psi$ factor. Our operator $T_{a}$ is linked to $\widetilde{T_{a}}$ by $T_{a} u=$ $\widetilde{T_{a}}\left(\psi\left(D_{x}\right) u\right)$, where $\psi\left(D_{x}\right)$ is the Fourier multiplier of symbol $\psi$. The operator $\widetilde{T_{a}}$ enjoys the same continuity properties as $T_{a}$, namely, for $a$ bounded it acts continuously $H^{s} \rightarrow H^{s}$ (Proposition 5.2.1 in [Mét08]).

1. We have $u v-T_{u} v-T_{v} u=u v-\widetilde{T_{u}} v-\widetilde{T_{v}} u+\widetilde{T_{u}}\left(1-\psi\left(D_{x}\right) v\right)+\widetilde{T_{v}}\left(1-\psi\left(D_{x}\right) u\right)$. Since $1-\psi(\xi)$ is compactly supported, $\widetilde{T_{u}}\left(1-\psi\left(D_{x}\right) v\right) \in H^{\infty}$. As $s, r>d / 2$, $u$ and $v$ are bounded and thus $\widetilde{T}_{u}, \widetilde{T_{v}}$ are continuous $H^{s+r-d / 2} \rightarrow H^{s+r-d / 2}$. We are reduced to proving $\left\|u v-\widetilde{T_{u}} v-\widetilde{T_{v}} u\right\|_{H^{s+r-d / 2}} \lesssim\|u\|_{H^{s}}\|v\|_{H^{r}}$, which is standard. We sketch the argument for completeness: set

$$
u=\sum_{j \geq 0} u_{j}, v=\sum_{j \geq 0} v_{j}
$$

Copyright $@$ by SIAM. Unauthorized reproduction of this article is prohibited. 
the nonhomogeneous Littlewood-Paley decomposition of $u, v$. Then $u v-$ $\widetilde{T_{u}} v-\widetilde{T_{v}} u=\sum_{|j-k| \leq 2} u_{j} v_{k}$ (this is a consequence of our choice for the function $\chi$ ), and the Fourier transform of $u_{j} v_{k}$ is supported in a ball of radius $C 2^{j}$. By Proposition 4.1.12 in [Mét08] we only have to prove $2^{j(s+r-n / 2)}\left\|u_{j} v_{k}\right\|_{L^{2}}$ $1_{|j-k| \leq 2} \in l^{2}\left(\mathbb{N}^{2}\right)$.

According to Bernstein inequalities (Corollary 4.1.7 in [Mét08]), $\left\|u_{j}\right\|_{L^{\infty}} \lesssim$ $2^{j(d / 2-s)}\left\|u_{j}\right\|_{H^{s}},\left\|v_{j}\right\|_{L^{2}} \lesssim 2^{-j r}\left\|v_{j}\right\|_{H^{r}}$ so that

$$
|k-j| \leq 2 \Rightarrow\left\|u_{j} v_{k}\right\|_{L^{2}} \lesssim 2^{j(d / 2-s-r)}\|u\|_{H^{s}}\left\|v_{k}\right\|_{H^{r}},
$$

and the conclusion follows from $\sum\left\|v_{j}\right\|_{H^{r}}^{2} \lesssim\|v\|_{H^{r}}^{2}$.

2. As previously, we write

$$
a u-T_{a} u=a u-\widetilde{T_{a}} u+\widetilde{T_{a}}\left(\left(1-\psi\left(D_{x}\right)\right) u\right),
$$

but $\left(1-\psi\left(D_{x}\right)\right) u \in H^{\infty}$, so that the second term in the right-hand side has its $H^{s}$ norm controlled by $\|a\|_{L^{\infty}}\|u\|_{L^{2}}$, while Proposition 5.2.2 in [Mét08] gives

$$
\left\|a u-\widetilde{T_{a}} u\right\|_{H^{s}} \lesssim\|\nabla a\|_{H^{s-1}}\|u\|_{L^{\infty}} .
$$

3. Again, we are reduced to estimating $a \partial_{x} u-\widetilde{T_{a}} \partial_{x} u$, which is done in Theorem 5.2.9 in [Mét08]. The result stated in the theorem is slightly different, but the proof gives the estimate needed.

Corollary 2.7. Let $s>d / 2, r \geq s+1, u \in H^{s}$, and $a \in H^{r}$ (resp., $a \in$ $\left.L^{\infty}, \nabla a \in H^{r-1}\right)$. We have $a u=T_{a} u+Q(u, a)$ with $\|Q(u, a)\|_{H^{r}} \lesssim\|u\|_{H^{s}}\|a\|_{H^{r}}$ (resp., $\left.\|u\|_{H^{s}}\left(\|\nabla a\|_{H^{r-1}}+\|a\|_{L^{\infty}}\right)\right)$.

Proof. The second case is a weaker version of the second point in the previous proposition.

For the first case, we have $a u=T_{a} u+T_{u} a+R(a, u)$, where $R$ already satisfies the estimate. But since (by Sobolev embedding) $u \in L^{\infty}$, Proposition 2.5 implies that $T_{u} a \in H^{r}$ with the expected estimate.

We denote by $[A, B]$ the commutator $A B-B A$ of two operators.

Proposition 2.8 (functional calculus). If $(a, b) \in \Gamma_{r}^{m} \times \Gamma_{s}^{n}, r, s \geq 1$, we have the composition rule $T_{a} T_{b}=T_{a \sharp b}+R$, where

$$
a \sharp b=\sum_{|\alpha|<\min (r, s)} \frac{1}{\alpha ! i^{|\alpha|}} \partial_{\xi}^{\alpha} a \partial_{x}^{\alpha} b,
$$

and $R$ is continuous $H^{t+m+n} \rightarrow H^{t+\min (r, s)}$. In particular,

- $\left[T_{a}, T_{b}\right]$ is continuous $H^{t+m+n-1} \rightarrow H^{t}$;

- if moreover $r, s \geq 2,\left[T_{a}, T_{b}\right]=T_{c}+R$, where $R$ is continuous $H^{t+m+n-2} \rightarrow$ $H^{t}$ and $c$ is defined by

$$
c=\frac{1}{i}\{a, b\}:=\frac{1}{i}\left(\sum \partial_{\xi_{j}} a \partial_{x_{j}} b-\partial_{x_{j}} a \partial_{\xi_{j}} b\right) .
$$

There is also a symbolic calculus for adjoints: let $a \in \Gamma_{r}^{m}$ be a matrix valued symbol (i.e., each coefficient is a symbol of $\left.\Gamma_{r}^{m}\right)$; we use such operators in the obvious way $(A u)_{i}=\left(\sum A_{i j} u_{j}\right)_{i}$. Set

$$
b(x, \xi)=\sum_{|\alpha|<r} \frac{1}{i^{|\alpha|} \alpha !} \partial_{x}^{\alpha} \partial_{\xi}^{\alpha} a^{*},
$$

Copyright (? by SIAM. Unauthorized reproduction of this article is prohibited. 
where $a^{*}$ is the adjoint matrix of $a$, namely, if $a=\left(a_{i, j}\right), a_{i, j}^{*}=\overline{a_{j, i}}$. Then $\left(T_{a}\right)^{*}-T_{b}$ is continuous $H^{s+m-r} \rightarrow H^{s}$. In particular for $r \geq 1,\left(T_{a}\right)^{*}=T_{a^{*}}+R$ with $R$ continuous $H^{s+m-1} \rightarrow H^{s}$.

Proof. The results are proved in [Mét08] at Theorems 6.1.1 and 6.2.1 for operators $\widetilde{T_{a}}$. But with our definition $T_{a}=\widetilde{T}_{a(x, \xi) \psi(\xi)}$ and $\nabla \psi$ is compactly supported. Thus it only adds in the formulas (2.2), (2.3) symbols which have compact support in $\xi$, which correspond to neglectable infinitely smoothing operators.

Combining the Sobolev embeddings with the Proposition 2.8, we get the following corollary.

Corollary 2.9. If $(a, b) \in H^{s} \times H^{r}, s, r>d / 2+1, T_{a} T_{b}=T_{a b}+R$, where $R$ is continuous $H^{t} \rightarrow H^{t+1}$.

If $a \in L^{\infty}, \nabla a \in H^{s}, b \in \Gamma_{r}^{m}, r \geq 2$, then $\left[T_{a}, T_{b}\right]=T_{\{a, b\}}+R$, where $R$ is continuous $H^{t+m} \rightarrow H^{t+2}$.

Proposition 2.10 (elliptic estimate). Let $a \in \Gamma_{1}^{m}$ be a symbol satisfying

$$
\operatorname{Re} a(x, \xi) \geq c(1+|\xi|)^{m} ;
$$

there are constants $C_{1}, C_{2}$ such that $\forall u \in H^{m}$,

$$
\|u\|_{H^{s}} \leq C_{1}\left\|T_{a} u\right\|_{H^{s-m}}+C_{2}\|u\|_{L^{2}} .
$$

Proof. In [Mét08, section 6.3] it is proved that

$$
\|u\|_{H^{s}} \leq C_{1}\left\|T_{a} u\right\|_{H^{s-m}}+C_{2}\|u\|_{H^{s-1}},
$$

which gives the result by using that for any $\varepsilon>0,\|u\|_{H^{s-1}} \leq \varepsilon\|u\|_{H^{s}}+C_{\varepsilon}\|u\|_{L^{2}}$.

3. The one-dimensional case. In this section we prove Kato smoothing in dimension 1. Several technical points are simplified in this case; in particular, we do not need further assumption than the existence of $(\rho, u)$ solution of (EK1D). The main result of this section is the following regularization for the solutions of (EK1D).

Theorem 3.1. Let $\left(\rho_{0}, u_{0}\right) \in L^{\infty} \times H^{s}$ such that $\partial_{x} \rho_{0} \in H^{s}, s>3 / 2$. Let $(\rho, u) \in C_{t} L^{\infty} \times C_{t} H^{s}$ be the solution of the Cauchy problem (EK1D)

$$
\left\{\begin{array}{l}
\partial_{t} \rho+\partial_{x}(\rho u)=0, \\
\partial_{t} u+\left(u \cdot \partial_{x}\right) u+\partial_{x} g(\rho)=\partial_{x}\left(K(\rho) \partial_{x}^{2} \rho+\frac{1}{2} K^{\prime}(\rho)\left|\partial_{x} \rho\right|^{2}\right),
\end{array}\right.
$$

which satisfies $\left(u, \partial_{x} \rho\right) \in C_{t} H^{s} \cap C_{t}^{1} H^{s-2}$. Then for any $\varepsilon>0$, we have

$$
\left(\partial_{x} \rho, u\right) /\langle x\rangle^{(1+\varepsilon) / 2} \in L_{l o c, t}^{2} H_{x}^{s+1 / 2} .
$$

3.1. Paralinearization of the Euler-Korteweg equations. Since $\zeta=R(\rho)$ is a smooth diffeomorphism, according to Proposition 5.2 it is equivalent to prove smoothing for the extended variables $(w, u)$ or for $z=u+i w$. We start with (SB) and consider

$$
z \in C_{t} H^{s} \cap C_{t}^{1} H^{s-2}, \zeta \in L^{\infty}, \partial_{x} \zeta \in C_{t} H^{s} \cap C_{t}^{1} H^{s-2}
$$

with $s>1+1 / 2$ such that

$$
\partial_{t} z+u \partial_{x} z+i w \partial_{x} z+i \partial_{x}\left(a \partial_{x} z\right)=-g^{\prime}(\zeta) \operatorname{Re}(z), z=u+i v .
$$

The Cauchy theory of the Euler-Korteweg system (for example, see [BGDD07, Proposition 4.3]) ensures that the $C_{t} H^{s}$ norm of $z$ is controlled by the $H^{s}$ norm of the initial data $\left(u_{0}, w_{0}\right)$. 
Remark 3.2. This statement is not entirely true. In fact to be rigorous it should be noted that the constants involved also depend on $\left\|\rho_{0}\right\|_{\infty}$, a fact that we will not recall in the rest of the article.

According to Corollary 2.7 we have

$$
\begin{array}{r}
u \partial_{x} z=T_{u} \partial_{x} z+R_{1}, R_{1} \in C_{t} H^{s}, \\
w \partial_{x} z=T_{w} \partial_{x} z+R_{2}, R_{2} \in C_{t} H^{s}, \\
a \partial_{x} z=T_{a} \partial_{x} z+R_{3}, R_{3} \in C_{t} H^{s+1}, \\
\text { hence } \partial_{x}\left(a \partial_{x} z\right)=\partial_{x}\left(T_{a} \partial_{x} z\right)+R_{4}, R_{4} \in C_{t} H^{s} .
\end{array}
$$

Thus (SB) implies

$$
\partial_{t} z+T_{u} \partial_{x} z+T_{i w} \partial_{x} z+i \partial_{x}\left(T_{a} \partial_{x} z\right)=R, R \in C_{t} H^{s} .
$$

In order to reduce the analysis to the study of a Schrödinger equation satisfied by some distribution $Z_{s} \in L^{2}$, it is natural to apply the operator $T_{|\xi|^{s}}$ to the equations. Moreover, as we pointed in the introduction it is important to cancel out the "bad" term $T_{i w} \partial_{x} z$. This kind of issue is classically tackled for the one-dimensional Schrödinger equation by a change of gauge. The paradifferential calculus brings to this point a (relative) simplification of the calculus and avoids the loss of derivatives that this kind of transformation may induce.

Following [BGDD06] we look for a multiplier which has the form $T_{|\xi|^{s} \varphi_{s}}$, where $\varphi_{s}(x, t)$, to be determined, does not vanish and belongs to $C_{t} W^{r, \infty} \cap C_{1}^{t} W^{r-2, \infty}$ for some $r>2$.

According to Proposition 2.8, the commutators

$$
\left[T_{|\xi|^{s} \varphi_{s}}, T_{u} \partial_{x}\right],\left[T_{\left.|\xi|\right|^{s} \varphi_{s}}, T_{w} \partial_{x}\right], \text { and }\left[T_{|\xi|^{s} \varphi_{s}}, \partial_{t}\right]
$$

define continuous operators $H^{s} \rightarrow L^{2}$; moreover

$$
\left[T_{|\xi|^{s} \varphi_{s}(x)}, i \partial_{x} T_{a} \partial_{x}\right]=T_{\left\{|\xi|^{s} \varphi_{s}(x),-|\xi|^{2} a(x)\right\}}+T^{\prime},
$$

where $T^{\prime}$ is continuous $H^{s} \rightarrow L^{2}$ and $\{\cdot, \cdot\}$ is the Poisson bracket

$$
\begin{aligned}
\left\{|\xi|^{s} \varphi_{s}(x),-|\xi|^{2} a(x)\right\} & =\partial_{\xi}\left(|\xi|^{s} \varphi_{s}\right) \partial_{x}\left(-|\xi|^{2} a(x)\right)-\partial_{x}\left(|\xi|^{s} \varphi_{s}\right) \partial_{\xi}\left(-|\xi|^{2} a(x)\right) \\
& =|\xi|^{s} \xi\left(-s \varphi_{s} \partial_{x} a+2 a \partial_{x} \varphi_{s}\right) .
\end{aligned}
$$

In order to cancel out the main order term $T_{|\xi|^{s} \varphi_{s} w} i \partial_{x} z=T_{-|\xi|^{s} \xi \varphi_{s} w} z$, it is therefore sufficient to have $-s \varphi_{s} \partial_{x} a+2 a \partial_{x} \varphi_{s}=\varphi_{s} w$. The choice $\varphi_{s}=\sqrt{\rho} a^{s / 2}$ gives

$$
\begin{aligned}
2 a \partial_{x}\left(a^{s / 2} \sqrt{\rho}\right) & =s a^{s / 2} \sqrt{\rho} \partial_{x} a+2 a^{s / 2+1} \frac{\partial_{x} \rho}{2 \sqrt{\rho}} \\
& =s \varphi_{s} \partial_{x} a+a^{s / 2} \frac{\rho w}{\sqrt{\rho}} \\
& =s \varphi_{s} \partial_{x} a+\varphi_{s} w .
\end{aligned}
$$

We define $Z_{s}=T_{\varphi_{s}|\xi|} z$. According to the commutator estimates and the previous computations the equation on $Z_{s}$ is

$$
\partial_{t} Z_{s}+T_{u} \partial_{x} Z_{s}+i \partial_{x}\left(T_{a} \partial_{x} Z_{s}\right)=R, R \in C_{t} L^{2},
$$

where the norm of $R$ is controlled by $\|(u, w)\|_{H^{s}} \lesssim\left\|\left(u_{0}, w_{0}\right)\right\|_{H^{s}}$. 
By ellipticity of $T_{\varphi_{s}|\xi|^{s}}$, it is clear that a gain of derivative for $z /\langle x\rangle^{(1+\varepsilon) / 2}$ is equivalent to a gain of derivatives for $Z_{s} /\langle x\rangle^{(1+\varepsilon) / 2}$. (Note that it relies on the fact that $\langle x\rangle^{-(1+\varepsilon) / 2}$ is smooth to make it commute with $T_{\varphi_{s}|\xi|^{s}}$.) Thus we have proved the following.

LEMma 3.3. The proof of Kato smoothing for $(u, v)$ amounts to proving

$$
\left\|Z_{s} /\langle x\rangle^{(1+\varepsilon) / 2}\right\|_{L_{t, l o c}^{2} H^{1 / 2}} \leq C\left\|Z_{s}\right\|_{C_{t} L^{2}}
$$

for $Z_{s}$ solution of

$$
\partial_{t} Z_{s}+T_{u} \partial_{x} Z_{s}+i \partial_{x}\left(T_{a} \partial_{x} Z_{s}\right)=R, R \in C_{t} L^{2}
$$

3.2. Smoothing effect on $Z_{s}$. Doi's method [Doi96] is based on estimates for

$$
\frac{d}{d t}\left\langle T_{p} Z_{s}, Z_{s}\right\rangle=\frac{d}{d t} \int_{\mathbb{R}}\left(T_{p} Z_{s}\right) \overline{Z_{s}} d x
$$

where $p(x, \xi)$ is a symbol chosen such that this derivative is bounded from below (up to neglectible terms) by $\left\|f Z_{s}\right\|_{H^{1 / 2}}$ for some $f$ decaying fast enough. Here we have

$$
\begin{aligned}
\frac{d}{d t}\left\langle T_{p} Z_{s}, Z_{s}\right\rangle= & \left\langle\partial_{t}\left(T_{p} Z_{s}, Z_{s}\right)\right\rangle+\left\langle T_{p} Z_{s}, \partial_{t} Z_{s}\right\rangle \\
= & -\left\langle T_{p} T_{u} \partial_{x} Z_{s}+i T_{p} \partial_{x} T_{a} \partial_{x} Z_{s}, Z_{s}\right\rangle \\
& -\left\langle T_{p} Z_{s}, u \partial_{x} Z_{s}+i \partial_{x} T_{a} \partial_{x} Z_{s}\right\rangle+R \\
= & \left\langle-\left[T_{p}, T_{u} \partial_{x}\right] Z_{s}-i\left[T_{p}, \partial_{x} T_{a} \partial_{x}\right] Z_{s}, Z_{s}\right\rangle+R \\
= & \left\langle T_{\left\{i p,|\xi|^{2} a\right\}} Z_{s}, Z_{s}\right\rangle+R,
\end{aligned}
$$

where $R \in L_{\text {loc }, t}^{1}$ (with a control by $\left\|Z_{s}\right\|_{C_{t} L^{2}}^{2}$ ).

Proposition 3.4. For any $\varepsilon>0$, there exists a symbol $p \in \Gamma_{\infty}^{0}$ such that

$$
\left\{i p,|\xi|^{2} a\right\} \geq c|\xi| /\langle x\rangle^{1+\varepsilon} \text {. }
$$

The construction of $p$ is classical and postponed to the appendix. Assume that such a $p$ exists. By integrating from 0 to $T$ and applying the weighted Gårding inequality of Appendix B we find

$$
\left\langle T_{p} Z_{s}(T), Z_{s}(T)\right\rangle-\left\langle T_{p} Z_{s}(0), Z_{s}(0)\right\rangle \geq \int_{0}^{T}\left\|Z_{s} /\langle x\rangle^{(1+\varepsilon) / 2}\right\|_{H^{1 / 2}}^{2}-C\left\|Z_{s}\right\|_{L^{2}}^{2} d t,
$$

so that

$$
\left\|Z_{s} /\langle x\rangle^{(1+\varepsilon) / 2}\right\|_{L_{l o c, t}^{2} H^{1 / 2}} \lesssim\left\|Z_{s}(0)\right\|_{L^{2}} \lesssim\left\|\left(u_{0}, w_{0}\right)\right\|_{H^{s}}
$$

The proof of this estimate is rigorous only for smooth initial data, namely, $Z_{s} \in$ $H^{2}$, since in this case the existence theorem of [BGDD06] ensures that $\frac{d}{d t}\left\langle T_{p} Z_{s}^{n}, Z_{s}^{n}\right\rangle$ belongs to $C_{t}$. If $Z_{s}$ belongs to $L^{2}$, we need to use smoothed initial data $Z_{s}(0)^{n} \in H^{2}$ that converge to $Z_{s}(0)$ in $L^{2}$. The $Z_{s}^{n}(t)$ satisfy the estimate (3.1), and we can pass to the limit in $n$ by using Fatou's lemma in both the left-and the right-hand side (up to using a subsequence such that $Z_{s}^{n}(t)$ converges almost everywhere to $Z_{s}(t)$ ).

This implies the following proposition, which implies Theorem 3.1.

Proposition 3.5. If $Z_{s}(0)$ belongs to $L^{2}$, then $Z_{s} /\langle x\rangle^{(1+\varepsilon) / 2}$ belongs to $L_{l o c, t}^{2} H_{x}^{1 / 2}$ for $\varepsilon$ arbitrarily small, and its norm is controlled by $\left\|\left(u_{0}, w_{0}\right)\right\|_{H^{s}}$.

Copyright (c) by SIAM. Unauthorized reproduction of this article is prohibited. 
4. Multidimensional case. In the case of several space variables, the degeneracy of the system may prevent the dispersive smoothing effect. To illustrate this fact one may look at the linearized constant coefficient system

$$
\partial_{t} z+\underline{u} \cdot \nabla z+i \nabla(\underline{a} \operatorname{div} z)=-\underline{g^{\prime}} w .
$$

If $z$ is divergence free this becomes a transport equation, for which there cannot be any smoothing. On the other hand, if $z$ is potential, the equation satisfied by $z$ is

$$
\partial_{t} z+\underline{u} \cdot \nabla z+i \underline{a} \Delta z=-\underline{g^{\prime}} w
$$

and by adapting the classical proof of Constantin and Saut [CS88] in the case of the Schrödinger equation one easily obtains the local gain of $1 / 2$ (spatial) derivative for the solutions.

Based on this simple example, we focus on the case where $z$ is potential, and we prove the same 1/2 Kato smoothing under a few standard additional assumptions (namely, flatness at infinity and nontrapping of the bicharacteristics). We recall that the multidimensional extended Euler-Korteweg system reads for the complex vector $z=u+i w$

$$
\partial_{t} z+(u \cdot \nabla) z+i(\nabla z) \cdot w+i \nabla(a \operatorname{div} z)=-g^{\prime}(\zeta) w .
$$

(Note that this reformulation uses the fact that $w$ is potential.) As previously, we reduce the problem to an $L^{2}$ estimate by using the new quantity $Z_{s}=T_{\varphi_{s}|\xi| s} z, \varphi_{s}$ being chosen so that commutators may control the "bad" term $i \nabla z \cdot w$. In fact, in the irrotational case we will find out that the previous choice $\varphi_{s}=\sqrt{\rho} a^{s / 2}:=\varphi_{0} a^{s / 2}$ still works. Our result reads as follows.

THEOREM 4.1. Under the following assumptions,

- $u_{0}$ is irrotational,

- the Hamiltonian $a(x, 0)|\xi|^{2}$ has no trapped bicharacteristic,

- $a(x, t) \in C_{t} W^{2, \infty} \cap C_{t}^{1} W^{1, \infty},\left|\partial_{t} a\right|+\left|\partial_{t} \nabla a\right|+|\nabla a| \leq C /\langle x\rangle^{1+\varepsilon}$;

then there exists $T>0$ such that

$$
\left\|(u, w) /(1+|x|)^{1+\varepsilon}\right\|_{L^{2}\left([0, T] ;\left(H^{s+1 / 2}\right)^{2 d}\right)} \lesssim\left\|u_{0}, w_{0}\right\|_{H^{s}} .
$$

Remark 4.2.

- Unlike the one-dimensional case, the time interval $[0, T]$ on which there is smoothing may be smaller than the lifespan of the solution. This is due to the fact that the nontrapping assumption is only locally (in time) true, and it is not clear whether it is "propagated."

- In our proofs we will use the fact that if $u_{0}$ is irrotational, then $z=u+$ $i w$ is irrotational. This follows from the fact that $w$ is a gradient and the implication $\operatorname{curl} u_{0}=0 \Rightarrow \operatorname{curl} u=0$ (Corollary 4.1 in [BGDD07]).

- Since $w=\sqrt{K(\rho) / \rho} \nabla \rho$, the same theorem holds if one replaces $w$ (resp., $\left.w_{0}\right)$ by $\nabla \rho$ (resp., $\nabla \rho_{0}$ ). (This is a mere consequence of Proposition 5.2 on product rules in Sobolev spaces.)

4.1. Paralinearization. We use the convention that $R$ is a generic harmless term in $C_{t} L_{x}^{2}$, controlled by $\left\|\left(u_{0}, w_{0}\right)\right\|_{H^{s}}$.

If $v: \mathbb{R}^{d} \times \mathbb{R}^{d} \rightarrow \mathbb{R}^{d}$, we write $T_{v} \cdot z=\sum_{j=1}^{d} T_{v_{j}} z_{j}, T_{v} \cdot \nabla z=\left(\sum_{j} T_{u_{j}} \partial_{j} z_{i}\right)_{i=1 \cdots d}$, and for $A: \mathbb{R}^{d} \times \mathbb{R}^{d} \rightarrow \mathbb{R}^{d^{2}}, T_{A} z:=\left(\sum_{j=1}^{d} T_{A_{i, j}} z_{j}\right)_{i=1 \cdots d}$. More generally when $A$ and 
$B$ are matrices valued with compatible dimensions, we write $T_{A} \cdot B=\left(\sum_{j} T_{A_{i, j}} B_{j, k}\right)_{i, k}$, the dot being omitted if one of the matrices is of size $1 \times 1$. The scalar rules of paradifferential calculus extend straightforwardly to this frame.

Following the existence theorem of [BGDD07], we assume that $z \in C_{t} H^{s} \cap$ $C_{t}^{1} H^{s-2}, \rho \in L^{\infty}$, has a positive lower bound and $\nabla \rho \in C_{t} H^{s}$ for some $s>1+d / 2$. In particular we have as in the previous section

$$
\begin{array}{r}
u \cdot \nabla z=T_{u} \cdot \nabla z+R_{1}, R_{1} \in C_{t} H^{s}, \\
w \cdot \nabla z=T_{w} \cdot \nabla z+R_{2}, R_{2} \in C_{t} H^{s}, \\
a \partial_{j} z=T_{a} \partial_{j} z+R_{3}, R_{3} \in C_{t} H^{s+1}, \\
\text { hence } \partial_{k}\left(a \partial_{j} z\right)=\partial_{k}\left(T_{a} \partial_{k} z\right)+R_{4}, R_{4} \in C_{t} H^{s} .
\end{array}
$$

We first exploit the curl-free assumption: let $Q$ be the projector on curl-free vector fields; its symbol is $\xi \xi^{t} /|\xi|^{2}$ and it satisfies $\nabla \operatorname{div} Q=\Delta Q$. This implies

$$
\nabla(a \operatorname{div} z)=a \nabla \operatorname{div} z+(\nabla a) \operatorname{div} z=\operatorname{div}(a \nabla z)+(\nabla a) \cdot \operatorname{div} z-\nabla z \cdot \nabla a .
$$

As in the previous section we take $\varphi_{s}=\sqrt{\rho} a^{s / 2}$ and we multiply (4.1) by $T_{\varphi_{s}|\xi|^{s}}$. This gives

$$
\begin{array}{r}
T_{\varphi_{s}|\xi|^{s}} \partial_{t} z+T_{\varphi_{s}|\xi|^{s}}(u \cdot \nabla z)+i T_{\varphi_{s}|\xi|^{s}}((\nabla z) \cdot w)+T_{\varphi_{s}|\xi|^{s}}(i \operatorname{div}(a \nabla z)) \\
+i T_{\varphi_{s}|\xi|^{s}}((\nabla a) \operatorname{div} z-\nabla z \cdot \nabla a)=R,
\end{array}
$$

hence $\partial_{t} Z_{s}+(u \cdot \nabla) Z_{s}+i\left(\nabla Z_{s}\right) \cdot w+i \operatorname{div}\left(a \nabla Z_{s}\right)+i\left((\nabla a) \operatorname{div} Z_{s}-\nabla Z_{s} \cdot \nabla a\right)$

$$
=R+\left[i \operatorname{div} a \nabla, T_{\varphi_{s}|\xi|}\right] z \text {. }
$$

Using the rules of paradifferential calculus we have

$$
\begin{aligned}
{\left[i \operatorname{div}(a \nabla \cdot), T_{\varphi_{s}|\xi|^{s}}\right] z } & =\left[T_{-i a|\xi|^{2}}, T_{\varphi_{s}|\xi|^{s}}\right] z+R \\
& =T_{\left\{-a|\xi|^{2}, \varphi_{s}|\xi|^{s}\right\}} z+R .
\end{aligned}
$$

But

$$
\begin{aligned}
\left\{-a|\xi|^{2}, \varphi_{s}|\xi|^{s}\right\} & =-2|\xi|^{s} a \xi \cdot \nabla \varphi_{s}+s|\xi|^{s} \varphi_{s} \nabla a \cdot \xi \\
& =-\varphi_{s}|\xi|^{s} \xi \cdot w
\end{aligned}
$$

so that

$$
\left[i \operatorname{div} a \nabla, T_{\varphi_{s}|\xi|^{s}}\right] z=i T_{w} \cdot \nabla Z_{s}+R=i \nabla Z_{s} \cdot w+R
$$

since $w$ is curl-free. The paralinearized equation on $Z_{s}$ is thus

$$
\partial_{t} Z_{s}+T_{u} \nabla Z_{s}+i \operatorname{div}\left(T_{a} \nabla Z_{s}\right)+i T_{\nabla a} \operatorname{div} Z_{s}-T_{(\xi \cdot) \nabla a} Z_{s}=R,
$$

where

$$
T_{(\xi) \nabla a} Z_{s}:=\left(\sum_{j=1}^{d}-i T_{\partial_{x_{j}} a} \partial_{x_{i}} Z_{j}\right)_{1 \leq i \leq d}=i\left(T_{\nabla a} \cdot\left(\nabla Z_{s}\right)^{t}\right)^{t},
$$

Remark 4.3. The fact that we replaced $\nabla(a \operatorname{div} \cdot)$ by $\operatorname{div}(a \nabla \cdot)$ has allowed the commutator $\left[i \operatorname{div} a \nabla, T_{\varphi_{s}|\xi| s}\right] z$ to cancel the "bad" term $i \nabla z \cdot w$. Without the curl free assumption there would have been a remaining term $i \nabla z \cdot w-i \operatorname{div}(z) w$.

Copyright $@$ by SIAM. Unauthorized reproduction of this article is prohibited. 
To summarize we have obtained the next lemma.

LEMma 4.4. The proof of Kato smoothing for $(u, v)$ amounts to proving

$$
\left\|Z_{s} /\langle x\rangle^{(1+\varepsilon) / 2}\right\|_{L^{2}\left([0, T] ; H^{1 / 2}\right)} \leq C\left\|Z_{s}\right\|_{C\left([0, T] ; L^{2}\right)}
$$

for $Z_{s}$ solution of

$$
\partial_{t} Z_{s}+T_{u} \cdot \nabla Z_{s}+i \operatorname{div}\left(T_{a} \nabla Z_{s}\right)+i T_{\nabla a} \operatorname{div} Z_{s}-T_{(\xi \cdot) \nabla a} Z_{s}=R
$$

with $R \in C\left([0, T] ; L^{2}\right)$ whose norm is controlled by $\left\|\left(u_{0}, w_{0}\right)\right\|_{H^{s}}$.

4.2. The smoothing effect. Let $p \in \Gamma_{\infty}^{0}$ a scalar symbol to be determined later, independent of time. Following Doi we differentiate with respect to $t$ the integral $\int \overline{Z_{s}} \cdot\left(T_{p} Z_{s}\right) d x:=\left\langle T_{p} Z_{s}, Z_{s}\right\rangle$

$$
\begin{aligned}
\frac{d}{d t}\left\langle T_{p} Z_{s}, Z_{s}\right\rangle= & \left\langle T_{p} \partial_{t} Z_{s}, Z_{s}\right\rangle+\left\langle T_{p} Z_{s}, \partial_{t} Z_{s}\right\rangle \\
= & \left\langle T_{p}\left(-\left(T_{u} \cdot \nabla\right) Z_{s}-i \operatorname{div}\left(a \nabla Z_{s}\right)+T_{\left((\xi \cdot) \nabla a-\nabla a \cdot \xi^{t}\right)} Z_{s}\right), Z_{s}\right\rangle \\
& +\left\langle T_{p} Z_{s},-\left(T_{u} \cdot \nabla\right) Z_{s}-i \operatorname{div}\left(a \nabla Z_{s}\right)+T_{\left((\xi) \nabla a-\nabla a \cdot \xi^{t}\right)} Z_{s}\right\rangle .
\end{aligned}
$$

Now using that $\operatorname{div}(a \nabla \cdot)$ is self-adjoint and $T_{i u \xi}^{*}=-T_{i u \xi}+Q$ with $Q$ of order 0 we find

$$
\begin{aligned}
\frac{d}{d t}\left\langle T_{p} Z_{s}, Z_{s}\right\rangle= & \left\langle-\left[T_{p}, i \operatorname{div} a \nabla\right] Z_{s}, Z_{s}\right\rangle+\left\langle-\left[T_{p}, T_{u} \cdot \nabla\right] Z_{s}, Z_{s}\right\rangle \\
& +\left\langle\left(T_{p} T_{\left((\xi \cdot) \nabla a-\nabla a \cdot \xi^{t}\right)}-\left(T_{\left((\xi \cdot) \nabla a-\nabla a \cdot \xi^{t}\right)}\right)^{*} T_{p}\right) Z_{s}, Z_{s}\right\rangle+I,
\end{aligned}
$$

where $I \in L_{\text {loc }, t}^{1}$ is controlled by $\left\|Z_{s}\right\|_{C_{t} L_{x}^{2}}^{2} \lesssim\left\|\left(u_{0}, w_{0}\right)\right\|_{H^{s}}^{2}$.

We will generically call such terms $I$ even though they may change from one line to another.

The commutator $\left[T_{p}, T_{u} \cdot \nabla\right]$ is an operator of order 0 ; thus $\left\langle-\left[T_{p}, T_{u} \cdot \nabla\right] Z_{s}, Z_{s}\right\rangle$ can be included in I. There is no such cancellation for the second line of $(4.3)$, since it is easily checked that $\left.T_{\left((\xi \cdot) \nabla a-\nabla a \xi^{t}\right)}\right)^{*}=T_{\left({ }_{\left.(\xi \cdot) \nabla a-\nabla a \xi^{t}\right)}\right.}+Q$ with $Q$ of order 0 . On the other hand, if we denote $Z_{j}$ the coordinates of the vector $Z_{s}$, we have

$$
\begin{aligned}
\operatorname{Re}\left(\left\langle T_{p} T_{(\xi \cdot) \nabla a-\nabla a \xi^{t}} Z_{s}, Z_{s}\right\rangle\right) & =\operatorname{Re} i \sum_{k, l}\left\langle T_{p}\left(T_{\partial_{k} a} \partial_{l} Z_{k}-T_{\partial_{l} a} \partial_{k} Z_{k}\right), Z_{l}\right\rangle \\
= & -\operatorname{Im}\left(\sum_{k, l}-\left\langle T_{p} Z_{k}, T_{\partial_{k} a} \partial_{l} Z_{l}\right\rangle-\left\langle T_{\partial_{l} a} \partial_{k} Z_{k}, T_{\bar{p}} Z_{l}\right\rangle\right)+I
\end{aligned}
$$

If $p$ is real we obtain by reordering the sum

$$
\begin{aligned}
\operatorname{Re}\left(\left\langle T_{p} T_{(\xi \cdot) \nabla a-\nabla a \cdot \xi^{t}} Z_{s}, Z_{s}\right\rangle\right)= & \operatorname{Im}\left(\sum_{k, l}\left\langle T_{p} Z_{l}, T_{\partial_{l} a} \partial_{k} Z_{k}\right\rangle+\left\langle T_{\partial_{l} a} \partial_{k} Z_{k}, T_{p} Z_{l}\right\rangle\right) \\
& +I=I .
\end{aligned}
$$

Copyright $\odot$ by SIAM. Unauthorized reproduction of this article is prohibited. 
Injecting these identities in (4.3) gives

$$
\operatorname{Re}\left(\frac{d}{d t}\left\langle T_{p} Z_{s}, Z_{s}\right\rangle\right)=\operatorname{Re}\left(\left\langle-\left[T_{p}, i \operatorname{div}(a \nabla)\right] Z_{s}, Z_{s}\right\rangle\right)+I .
$$

We may now paralinearize the last term: $i \operatorname{div}(a \nabla \cdot)=-T_{i a|\xi|^{2}}+Q_{1}$ with $Q_{1}$ of order 1 ; thus

$$
\left\langle-\left[T_{p}, i \operatorname{div}(a \nabla \cdot)\right] Z_{s}, Z_{s}\right\rangle=\left\langle\left[T_{p}, T_{i a|\xi|^{2}}\right] Z_{s}, Z_{s}\right\rangle+I=\left\langle T_{\left\{p, a|\xi|^{2}\right\}} Z_{s}, Z_{s}\right\rangle .
$$

There is a huge gain here since we can now decouple the equations on $Z_{s}$ in equations for each of its coordinates $Z_{j}$ :

$$
\forall j=1 \cdots d, \operatorname{Re}\left(\frac{d}{d t}\left\langle T_{p} Z_{j}, Z_{j}\right\rangle\right)=\operatorname{Re}\left(\left(\left\langle T_{\left\{p, a|\xi|^{2}\right\}} Z_{j}, Z_{j}\right\rangle\right)+I .\right.
$$

We may now apply a paradifferential version of Doi's operator construction.

Lemma 4.5. Under the assumptions of Theorem 4.1, there exists a real valued symbol $p \in \Gamma_{1}^{0}$ and some constants $c, C>0$ such that for $0 \leq t \leq T$ small enough

$$
\left\{p, a(x, t)|\xi|^{2}\right\}:=\sum_{j=1}^{d}\left(\partial_{\xi_{j}} p\right) \partial_{x_{j}}\left(a|\xi|^{2}\right)-\left(\partial_{x_{j}} p\right) \partial_{\xi_{j}}\left(a|\xi|^{2}\right) \geq \frac{c|\xi|}{\langle x\rangle^{1+\varepsilon}}-C .
$$

The (sketch of) construction of this operator is made in Appendix C. The application of the weighted Gårding inequality of Proposition B.1 combined with Lemma 4.5 readily gives

$$
\begin{aligned}
\operatorname{Re}\left(\int_{0}^{T} \frac{d}{d t}\left\langle T_{p} Z_{s}, Z_{s}\right\rangle\right) & =\operatorname{Re}\left(\int_{0}^{T}\left\langle T_{\left\{p, a|\xi|^{2}\right\}} Z_{s}, Z_{s}\right\rangle\right)+\int_{0}^{T} I \\
& \geq c^{\prime}\left\|Z_{s} /\langle x\rangle^{(1+\varepsilon) / 2}\right\|_{H^{1 / 2}}^{2}-C\|Z\|_{L^{2}}^{2}+\int_{0}^{T} I .
\end{aligned}
$$

(Note that the real parts do not matter since up to harmless terms added in $I$ we may replace $T_{\left\{p, a|\xi|^{2}\right\}}$ by the self-adjoint operator $T_{\left\{p, a|\xi|^{2}\right\}}+T_{\left\{p, a|\xi|^{2}\right\}}^{*}$.) result:

By using regularized initial data as in the previous section, we obtain the expected

$$
\left\|Z_{s} /\langle x\rangle^{(1+\varepsilon) / 2}\right\|_{L^{2}\left([0, T] ; H^{1 / 2}\right)}^{2} \lesssim\left\|Z_{s}(0)\right\|_{L^{2}}^{2} \lesssim\left\|\left(u_{0}, w_{0}\right)\right\|_{H^{s}}^{2} .
$$

5. Solutions with nonzero endstates. The authors of [BGDD07] did not restrict their analysis to solutions $(u, w)$ vanishing at infinity; indeed, there exists special traveling waves solutions such that $u$ has different endstates. (They call such solutions capillary profiles.) Those solutions are physically pertinent (they can correspond to a change of state) and thus should be included in the present analysis. We will work under the regularity assumptions of Theorem 1.1 in [BGDD07] supplemented by the assumptions of our Theorem 4.1.

THEOREM 5.1. Let $(\underline{\rho}, \underline{u})$ be a smooth bounded solution to (EK) such that

$$
\left(\nabla^{2} \underline{\rho}, \nabla \underline{u}\right) \in C\left([0, T] ; H^{s+3}\right)
$$

and $\underline{u}$ is irrotational. We denote $\left(\underline{\rho_{0}}, \underline{u_{0}}\right):=(\underline{\rho}(t=0), \underline{u}(t=0))$. Let $\left(\rho_{0}, u_{0}\right)$ be a initial data such that

$$
\left(\rho_{0}, u_{0}\right)-\left(\underline{\rho_{0}}, \underline{u_{0}}\right) \in H^{s+1} \times H^{s}, s>1+d / 2 .
$$

Copyright (c) by SIAM. Unauthorized reproduction of this article is prohibited. 
We assume that

$\underline{\rho_{0}}\left([0, T] \times \mathbb{R}^{d}\right) \subset C$ with $C$ a compact set of $\mathbb{R}$ on which $K$ and $g_{0}$ are smooth.

$$
\left\{\begin{array}{l}
\partial_{t} \rho+\operatorname{div}(\rho u)=0 \\
\partial_{t} u+(u \cdot \nabla) u+\nabla g_{0}(\rho)=\nabla\left(K(\rho) \Delta \rho+\frac{1}{2} K^{\prime}(\rho)|\nabla \rho|^{2}\right), \\
\left.(\rho, u)\right|_{t=0}=\left(\rho_{0}, u_{0}\right) .
\end{array}\right.
$$

Let $(\rho, u) \in(\underline{\rho}, \underline{u})+C\left([0, T] ; H^{s+1} \times H^{s}\right)$ be the solution provided by Theorem 1.1 in [BGDD07]. Then, assuming that $(\rho-\rho, u-\underline{u})$ satisfies the assumptions of Theorem 4.1 , there exists $T^{\prime} \leq T$ such that the solution of (5.1) satisfies moreover

$$
(\nabla \rho, u)-(\nabla \underline{\rho}, \underline{u}) \in L^{2}\left(\left[0, T^{\prime}\right] ; H_{l o c}^{s+1 / 2}\right) \times H_{l o c}^{s+1 / 2} .
$$

Key ingredients for this result are standard multiplication and composition estimates in Sobolev spaces, which we recall here for the ease of the reader. Proofs can be found in Appendix B of [BGDD07].

Proposition 5.2. In every case, we assume $s \geq 0$. The product rule is as follows: For $k \in \mathbb{N}$, there exists $C(s, k, d)$ such that

$$
\|u v\|_{H^{s}} \leq C\left(\|u\|_{L^{\infty}}\|v\|_{H^{s}}+\left\|\nabla^{k} u\right\|_{H^{s-k}}\|v\|_{L^{\infty}}\right) .
$$

The composition rules are as follows: Let $F \in W^{\sigma+1, \infty}(I ; \mathbb{R})$, where $\sigma$ is the smallest integer such that $\sigma \geq s$, and assume $F(0)=0$. Then if $\operatorname{Im}(v) \subset \subset I$,

$$
\|F(u)\|_{H^{s}} \leq C\left(1+\|u\|_{L^{\infty}}\right)^{\sigma}\left\|F^{\prime}\right\|_{W^{\sigma, \infty}}\|v\|_{H^{s}} .
$$

Without assuming $F(0)=0$ and for $m \in \mathbb{N}^{*}$,

$$
\left.\| \nabla^{m} F(v)\right)\left\|_{H^{s}} \leq C\left(1+\|v\|_{L^{\infty}}\right)^{m+\sigma}\right\| F^{\prime}\left\|_{W^{m+\sigma, \infty}}\right\| D^{m} v \|_{H^{s}} .
$$

If $\operatorname{Im}(v) \subset \subset I, \operatorname{Im}(w) \subset \subset I$,

$$
\begin{aligned}
& \|F(w)-F(v)\|_{H^{s}} \leq C\left(\|F\|_{L^{\infty}}\|v-w\|_{H^{s}}\right. \\
& \quad+\left(1+\|v\|_{L^{\infty}}+\|w\|_{L^{\infty}}\right)^{\sigma+k+1}\left\|F^{\prime}\right\|_{W^{\sigma+k}}\left(\left\|\nabla^{k} v\right\|_{H^{s-k}}+\left\|\nabla^{k}(w-v)\right\|_{H^{s-k}}\right) .
\end{aligned}
$$

We may now prove Theorem 5.1.

Proof of Theorem 5.1. We focus on the extended system

$$
\partial_{t} z+(u \cdot \nabla) z+i(\nabla z) \cdot w+i \nabla(a \operatorname{div} z)=-g^{\prime}(\zeta) w .
$$

Set $\widetilde{u}=u-\underline{u}, \widetilde{w}=w-\underline{w}, \widetilde{z}=z-\underline{z}$; then $\widetilde{z}$ satisfies

$$
\begin{aligned}
\partial_{t} \widetilde{z}+u \cdot \nabla \widetilde{z}+i \nabla \widetilde{z} \cdot w+i \nabla(a \operatorname{div} \widetilde{z})= & -g^{\prime} w+\underline{g^{\prime}} \underline{w}-\widetilde{u} \cdot \nabla \underline{z}-i \nabla \underline{z} \cdot \widetilde{w} \\
& +i \nabla((a-\underline{a}) \operatorname{div} \underline{z}) \\
:= & R
\end{aligned}
$$

with $\underline{a}=a(\underline{\zeta})$.

We are mostly reduced to the argument of the previous section. Indeed, it is easy to see that the previous analysis can still be applied for the left-hand term. (Note

Copyright (C) by SIAM. Unauthorized reproduction of this article is prohibited. 
that the construction of $p$ essentially relies on the fact that $a$ is bounded away from zero, in particular the limits of $u$ at $\pm \infty$ do not matter, and the gauge transformation only relies on the relation between $a(\zeta)$ and $w$.) If we use as previously the function $\widetilde{Z_{s}}=T_{\varphi_{s}|\xi|} \widetilde{z}$, the only new terms in

$$
\frac{d}{d t}\left\langle T_{p} Z_{s}, Z_{s}\right\rangle
$$

are $\left\langle T_{p} R, Z_{s}\right\rangle$ and $\left\langle T_{p} Z_{s}, R\right\rangle$. In order to include these in $I \in L_{l o c, t}^{1}$, it suffices to check that $R \in C_{t} H^{s}$.

Since $\nabla \underline{z} \in C_{t} H^{s+1}$ and $\widetilde{z} \in C_{t} H^{s}$ the rules of product in Sobolev spaces imply $-\widetilde{u} \cdot \nabla \underline{z}-i \nabla \underline{z} \cdot \widetilde{w} \in C_{t} H^{s}$.

Now using the rules of composition we also have $a-\underline{a} \in C_{t} H^{s+1}$, which implies $i \nabla((a-\underline{a}) \operatorname{div} \underline{z}) \in C_{t} H^{s}$. Similarly, noting that $-g^{\prime} w+\underline{g^{\prime}} \underline{w}=\nabla(-g(\zeta)+g(\underline{\zeta}))$ we have $-p(\zeta)+p(\underline{\zeta}) \in C_{t} H^{s+1}$ (we see here that the assumption on the decay of the perturbation is essential even for $\widetilde{\rho}$ ), and thus $-g^{\prime} w+\underline{g^{\prime}} \underline{w} \in C_{t} H^{s}$. This allows us to neglect these new terms, and the rest of the proof goes as in section 4 .

Remark 5.3. The careful reader may have noted that we do not really need

$$
\left(\nabla^{2} \underline{\rho}, \nabla \underline{u}\right) \in C\left([0, T] ; H^{s+3}\right) .
$$

This is in fact necessary for the existence theorem in [BGDD07], but the Kato smoothing only requires $\left(\nabla^{2} \underline{\rho}, \nabla \underline{u}\right) \in C\left([0, T] ; H^{s+1}\right)$.

The decay assumption for the derivatives of $a$ are somehow not satisfying (nor satisfied!) in our frame because the Euler-Korteweg system admits special planar traveling waves solutions which only depend on $x \cdot \mathbf{n}-c t$ ( $\mathbf{n}$ is the direction of propagation, $c$ is the speed; see [BGDDJ05]). In particular, for $t$ fixed they are constant on any affine hyperplane orthogonal to $\mathbf{n}$ and $\nabla a(x \cdot \mathbf{n})$ can obviously not decay as $1 /\langle x\rangle^{1+\varepsilon}$. Though we do not have a general result, we will give some insights indicating that smoothing may occur also for perturbations of traveling waves. We restrict ourselves to the extended system linearized near a smooth traveling wave $(\underline{\zeta}, \underline{u}, \underline{w})$ of initial value $\left(\underline{\zeta_{0}}, \underline{u_{0}}, \underline{w_{0}}\right)$.

$$
\begin{cases}\partial_{t} z+\underline{u} \cdot \nabla z+i \nabla z \cdot \underline{w}+i \nabla(\underline{a} \operatorname{div} z) & =-\underline{g^{\prime} w}-\underline{g^{\prime \prime}} \zeta \underline{w}-z \cdot \nabla \underline{u} \\ \left.z\right|_{t=0}=z_{0}=u_{0}+i w_{0}, & -i \nabla \underline{z} \cdot w-i \nabla\left(\underline{a^{\prime}} \zeta \operatorname{div} \underline{z}\right),\end{cases}
$$

where we denote generic $\underline{f}=f(\underline{u}, \underline{w}, \underline{\zeta})$ and $\underline{f_{0}}=f\left(\underline{u_{0}}, \underline{w_{0}}, \underline{\zeta_{0}}\right)$. Up to a linear change of basis, we may assume that $\mathbf{n}=\left(\begin{array}{lllll}1 & 0 & \cdots & 0\end{array}\right)$ and we will use the notation $x=\left(x_{1}, x^{\prime}\right)$.

Proposition 5.4. The bicharacteristics

$$
\left\{\begin{array}{l}
\dot{X}\left(t, x_{0}, \xi_{0}\right)=2 a_{0}(X) \Xi, t \geq 0, \\
\dot{\Xi}\left(t, x_{0}, \xi_{0}\right)=-|\Xi|^{2} \nabla a_{0}(X), t \geq 0, \\
X\left(0, x_{0}, \xi_{0}\right)=x_{0}, \Xi\left(\overline{0,} x_{0}, \xi_{0}\right)=\xi_{0},
\end{array}\right.
$$

are not trapped, i.e., $\left|X\left(t, x_{0}, \xi_{0}\right)\right| \rightarrow|t| \rightarrow \infty \infty$. More precisely, they satisfy the following uniform nontrapping property:

$$
\begin{array}{r}
\left.\forall\left(x_{0}, \xi_{0}\right), \xi_{0} \neq 0, \exists r>0: \forall\left(x_{1}, x^{\prime}, \xi\right) \in\right] x_{0,1}-r, x_{0,1}+r\left[\times \mathbb{R}^{d-1} \times B\left(\xi_{0}, r\right):\right. \\
\forall K>0, \exists T_{K}>0: \forall|t| \geq T_{K}:|X(t, x, \xi)-x| \geq K .
\end{array}
$$


Proof. We first check "simple" nontrapping. Recall that the bicharacteristics are integral curves of the Hamiltonian $a_{0}(x)|\xi|^{2}$, whose conservation readily implies

$$
C / \sup a_{0} \leq|\Xi|^{2} \leq C / \inf a_{0} .
$$

Since $a_{0}=a_{0}\left(x_{1}\right)$, we have

$$
-|\Xi|^{2} \nabla a_{0}(X)=-|\Xi|^{2}\left(\begin{array}{c}
a_{0}^{\prime} \\
0 \\
\vdots \\
0
\end{array}\right) .
$$

As a consequence, $\Xi_{j}(t)=\xi_{0, j}$ and $\left(X_{j}\right)_{2 \leq j \leq d}$ are monotone of slope larger than $\inf \left(a_{0}\right)\left|\xi_{0, j}\right|$. They remain bounded in $t$ iff

$$
\Xi_{2}=\cdots=\Xi_{n}=0
$$

But in this case we have $a_{0}(X)|\Xi|^{2}=a_{0}\left(X_{1}\right)\left|\Xi_{1}\right|^{2}$; the conservation of this quantity implies that $\Xi_{1}$ is uniformly bounded away from 0 and in particular has constant sign. We deduce that $X_{1}(t)$ is monotone and (the modulus of) its slope has a lower bound, thus $\left|X_{1}(t)\right| \rightarrow_{t \rightarrow \infty} \infty$, and there can be no trapped bicharacteristic.

Since we have no decay assumption, uniform nontrapping cannot be deduced from the simple nontrapping. We give here a direct proof. Two cases must be distinguished:

- There exists $j \geq 2$ such that $\xi_{0, j} \neq 0$. For $r$ small enough,

$$
\forall(x, \xi) \in B\left(\left(x_{0}, \xi_{0}\right), r\right),\left|\xi_{j}\right| \geq\left|\xi_{0, j}\right| / 2,
$$

which directly implies $\left|X_{j}(s, x, \xi)-x_{j}\right| \geq\left|\xi_{0, j}\right| \inf \left(\underline{a_{0}}\right)|s|$ and thus the uniform nontrapping for this case.

- For $j \geq 2, \xi_{0, j}=0$. We denote $\xi^{\prime}=\left(\xi_{2}, \cdots, \xi_{d}\right)$. Then $\underline{a_{0}}(X)|\Xi|^{2}=$ $\underline{a_{0}}(X)\left(\left|\Xi_{1}\right|^{2}+\mid \xi_{2}^{2}+\cdots+\xi_{d}^{2}\right)=\underline{a_{0}}(x)|\xi|^{2}$. Take $r$ small enough such that $\left.\bar{\forall}(x, \xi) \in B\left(\left(x_{0}, \xi_{0}\right), r\right),\left|\xi^{\prime}\right|^{2}<\inf \underline{a_{0}}\right) /\left(2 \sup \left(\underline{a_{0}}\right)\right)\left|\xi_{1}\right|^{2}$. This gives

$$
\left|\Xi_{1}\right|^{2}+\left|\xi^{\prime}\right|^{2} \geq \frac{\inf \left(\underline{a_{0}}\right)}{\sup \left(\underline{a_{0}}\right)}|\xi|^{2} \Rightarrow\left|\Xi_{1}\right|^{2} \geq \frac{\inf \left(\underline{a_{0}}\right)}{2 \sup \left(\underline{a_{0}}\right)}\left|\xi_{1}\right|^{2} .
$$

As a consequence $\Xi_{1}$ has a constant sign, and up to decreasing $r$ so that $\left|\xi_{1}\right| \geq\left|\xi_{0,1}\right| / 2$ we find

$$
\left|X_{1}(s, x, \xi)-x_{1}\right| \geq \frac{\inf \left(\underline{\underline{a_{0}}}\right)^{3 / 2}}{\sqrt{2 \sup \left(\underline{a_{0}}\right.}}\left|\xi_{0,1}\right||s|,
$$

which implies again uniform nontrapping.

Uniform nontrapping is a key assumption for the construction of Doi's symbol $p$, and (to the author's opinion) it is deeper than the polynomial decay at infinity of $a$ (namely, $\left|\partial_{t} a\right|+\left|\partial_{t} \nabla a\right|+|\nabla a| \leq C /\langle x\rangle^{1+\varepsilon}$ ). Thus it seems reasonable to expect that smoothing occurs as soon as Property 5.4 is fulfilled without decay needed. We prove this now under a restriction on the graph of $a_{0}$.

Proposition 5.5. Assume that there exists $\alpha>0$ such that

$$
2 \sqrt{\underline{a_{0}}\left(x_{1}\right)}-\frac{a_{0}^{\prime}\left(x_{1}\right)}{\sqrt{\underline{a_{0}}\left(x_{1}\right)}} x_{1} \geq \alpha \text {. }
$$

Copyright (C) by SIAM. Unauthorized reproduction of this article is prohibited. 
Then there exists $T>0$ such that the solution $(u, w)$ of the linearized system (5.2) satisfies

$$
\forall \varepsilon>0, \exists T>0:\left\|(u, w) /\langle x\rangle^{1+\varepsilon}\right\|_{L^{2}([-T, T])\left(\left(H^{s+1 / 2}\right)^{2}\right)} \leq C\left\|\left(u_{0}, w_{0}\right)\right\|_{\left(H^{s}\right)^{2}} .
$$

Proof. The proof is essentially similar to that of Theorem 5.1; thus we will only detail the original points. By the same arguments it is reduced to the smoothing for $Z_{s}=T_{\varphi_{s}|\xi|^{s}} z=T_{\varphi_{s}|\xi|^{s}}(u+i w)$, which satisfies the equation

$$
\frac{d}{d t}\left\langle T_{p} Z_{s}, Z_{s}\right\rangle=\left\langle T_{p}\left(-i \nabla\left(\underline{a} \operatorname{div} Z_{s}\right)\right), Z_{s}\right\rangle+\left\langle T_{p} Z_{s},-i \nabla\left(\underline{a} \operatorname{div} Z_{s}\right)\right\rangle+I,
$$

where $I \in L_{\text {loc }, t}^{1}$ has a norm controlled by $\left\|\left.Z\right|_{t=0}\right\|_{L^{2}}$. It is sufficient to construct $p$ such that $\left\{\underline{a}|\xi|^{2}, p\right\} \geq|\xi| /\langle x\rangle^{1+\varepsilon}-C$. If there exists $q$ such that $\partial_{x}^{\alpha} \partial_{\xi}^{\beta} q \leq C\langle x\rangle\langle\xi\rangle^{-|\beta|}$ and $\left\{\underline{a}|\xi|^{2}, q\right\} \geq|\xi|$ the method to deduce $p$ from $q$ can be directly applied. (This step is indeed independent of the assumptions on $\underline{a_{0}}$.) It remains to prove that such a $q$ exists.

We take $q$ of the form $f\left(x_{1}-c t\right)\langle x, \xi\rangle /|\xi|$. Then

$$
\begin{aligned}
\left\{\underline{a}|\xi|^{2}, q\right\}= & 2 f\left(x_{1}-c t\right) \underline{a}\left(x_{1}-c t\right)|\xi|+2 a_{0} \xi_{1} f^{\prime}\left(x_{1}-c t\right) \frac{\langle x, \xi\rangle}{|\xi|} \\
& -\underline{a^{\prime}}\left(x_{1}-c t\right)|\xi| f\left(x_{1}-c t\right)+\underline{a}^{\prime}\left(x_{1}-c t\right) \frac{f\left(x_{1}-c t\right)}{|\xi|}\langle x, \xi\rangle \xi_{1} \\
= & |\xi|\left(2 \underline{a} f-x_{1} \underline{a^{\prime}} f\right)+\frac{\xi_{1}\langle x, \xi\rangle}{|\xi|}\left(\underline{a^{\prime}} f+2 f^{\prime} \underline{a}\right) .
\end{aligned}
$$

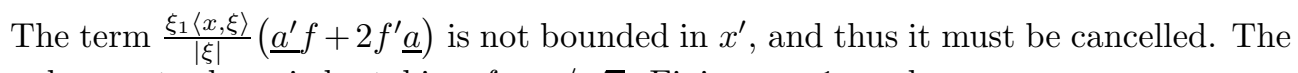
only way to do so is by taking $f=c / \sqrt{\underline{a}}$. Fixing $c=1$, we have

$$
\begin{aligned}
\left\{\underline{a}|\xi|^{2}, q\right\}=|\xi|\left(2 \underline{a} f-x_{1} \underline{a^{\prime}} f\right)=|\xi|\left(2 \sqrt{\underline{a}}-x_{1} \frac{\underline{a}^{\prime}}{\sqrt{\underline{a}}}\right) & \geq|\xi|\left(\alpha-c t \frac{\underline{a}^{\prime}}{\sqrt{\underline{a}}}\right) \\
& \geq \frac{\alpha|\xi|}{2}
\end{aligned}
$$

for $t$ small enough.

Remark 5.6. The condition

$$
2 \sqrt{\underline{a_{0}}\left(x_{1}\right)}-\frac{a_{0}^{\prime}\left(x_{1}\right)}{\sqrt{\underline{a_{0}}\left(x_{1}\right)}} x_{1} \geq \alpha
$$

may seem very artificial; it is, however, satisfied by several traveling waves. Obviously if (5.3) is true for $a_{0}(\cdot-b)$ instead of $a_{0}$, it then suffices to perform a shift of coordinates to apply Proposition 5.5, and thus the result only depends on the shape of $\underline{a_{0}}$ rather than its graph. In particular, provided $a_{0}$ has a convenient shape, the smoothing effect for the linearized system may be obtained on arbitrary time intervals by simply repeating the argument on small intervals.

The most obvious profiles satisfying this assumption are those such that $\underline{a_{0}}$ is increasing and then decreasing. Using

$$
2 \sqrt{\underline{\underline{a_{0}}}\left(x_{1}\right)}-\frac{a_{0}^{\prime}\left(x_{1}\right)}{\sqrt{\underline{a_{0}}}\left(x_{1}\right)} x_{1}=2 \sqrt{\underline{a_{0}}(0)}+\int_{0}^{x_{1}} \frac{a_{0}^{\prime}}{\sqrt{\underline{a_{0}}}}(y)-\frac{a_{0}^{\prime}}{\sqrt{\underline{a_{0}}}}\left(x_{1}\right) d y,
$$

Copyright (C) by SIAM. Unauthorized reproduction of this article is prohibited. 
we see that the case where $\sqrt{a_{0}}$ is increasing with an inflexion point at $x_{1}=0$ works, too. (See [BGDDJ05] for various examples of traveling profiles in dimension 1.)

\section{Appendix A. Construction of $p$ (one-dimensional case).}

Proposition A.1. For any $\varepsilon>0$, there exists $p \in \Gamma_{\infty}^{0}, c>0$ such that

$$
\left\{|\xi|^{2} a, i p\right\} \geq c|\xi| /\langle x\rangle^{1+\varepsilon} \text {. }
$$

Remark A.2. As was pointed out by the reviewer, one could simply check that the symbol $\xi /|\xi| \int_{0}^{x} 1 /\langle y\rangle^{1+\varepsilon} d y$ works; however, we emphasize that the construction of the following proof is essential for the multidimensional case.

Proof. The obvious candidate is $q(x, \xi)=x \frac{\xi}{|\xi|}$ since $\left\{|\xi|^{2} a, i x \frac{\xi}{|\xi|}\right\}=2 \xi \xi /|\xi| a=$ $2 a|\xi|$, but it is not in $\Gamma_{\infty}^{0}$ since it is not bounded in $x$. Thus it is necessary to use convenient truncations of this function. Let $\varphi \in C^{\infty}(\mathbb{R} ;[0,1])$ be such that $\varphi=$ $0, x \leq 1, \varphi=1, x \geq 2$. For $\delta>0$ (supposed small, specified later) we define $\varphi_{+}=\varphi(x / \delta), \varphi_{-}=\varphi(-x / \delta), \varphi_{0}=1-\varphi_{+}-\varphi_{-}$. If $\psi_{j}=\varphi_{j}(q /\langle x\rangle)$, we define the symbol $p$ by

$$
p(x, \xi)=\frac{q}{\langle x\rangle} \psi_{0}+(f(|q|)+2 \delta)\left(\psi_{+}-\psi_{-}\right),
$$

where $f$ is a primitive of $1 /\langle x\rangle^{1+\varepsilon}$.

Using the fact that $\xi /|\xi|$ is a constant function of $\xi$ outside 0 , we find

$$
\begin{aligned}
\left\{|\xi|^{2} a, i p\right\}= & \partial_{\xi}\left(a|\xi|^{2}\right) \partial_{x} p=2 \xi a\left(\psi_{0} \xi /(\langle x\rangle|\xi|)+q \partial_{x}\left(\psi_{0} /\langle x\rangle\right)\right) \\
& +2 a \xi \partial_{x}(f(q)+2 \delta) \psi_{+}+2 a \xi(f(q)+2 \delta) \partial_{x} \psi_{+} \\
& -2 a \xi \partial_{x}(f(q)+2 \delta) \psi_{-}-2 a \xi(f(q)+2 \delta) \partial_{x} \psi_{-} .
\end{aligned}
$$

We set

$$
\left\{\begin{array}{l}
I_{1}=2 \xi a\left(\psi_{0} \xi /(\langle x\rangle|\xi|)+q \partial_{x}\left(\psi_{0} /\langle x\rangle\right)\right) \\
I_{2}=2 a \xi \partial_{x}(f(q)+2 \delta) \psi_{+} \\
I_{3}=2 a \xi(f(q)+2 \delta) \partial_{x} \psi_{+} \\
I_{4}=-2 a \xi \partial_{x}(f(q)+2 \delta) \psi_{-} \\
I_{5}=-2 a \xi(f(q)+2 \delta) \partial_{x} \psi_{-}
\end{array}\right.
$$

The $I_{j}$ are handled as follows:

- First term: $I_{1}=2 a|\xi| \psi_{0} /\langle x\rangle+2 a \xi q \partial_{x}\left(\psi_{0} /\langle x\rangle\right)$ with

$$
\begin{aligned}
q \partial_{x}\left(\psi_{0} /\langle x\rangle\right) & =-\psi_{0} q x /\langle x\rangle^{3}+q /\langle x\rangle \psi_{0}^{\prime} \partial_{x}(x /\langle x\rangle \xi /|\xi|) \\
& =-\psi_{0} q x /\langle x\rangle^{3}+q \psi_{0}^{\prime} /\langle x\rangle^{4} \xi /|\xi| ;
\end{aligned}
$$

thus

$$
I_{1}=2 a|\xi| \psi_{0} /\langle x\rangle+2 a \xi\left(-\psi_{0} q x /\langle x\rangle^{3}+q \psi_{0}^{\prime} /\langle x\rangle^{4} \xi /|\xi|\right) .
$$

- Second term: $I_{2}=2 a|\xi| \operatorname{sgn}(q) f^{\prime}(|q|) \psi_{+}=2 a|\xi| /\langle x\rangle^{1+\varepsilon} \psi_{+}$.

- Third term: $I_{3}=2 a \xi(f(|q|)+2 \delta) \psi_{+}^{\prime} /\langle x\rangle^{3}$.

- Fourth term: $I_{4}=-2 a|\xi| \operatorname{sgn}(q) f^{\prime}(|q|) \psi_{-}=2 a|\xi| /\langle x\rangle^{1+\varepsilon} \psi_{-}$(since $\psi_{-}$vanishes for $q \geq 0$ ).

- Fifth term:

$$
I_{5}=-2 a \xi(f(|q|)+2 \delta) \psi_{-}^{\prime} /\langle x\rangle^{3}=2 a \xi\left(f(|q|+2 \delta) \psi_{+}^{\prime}(-x,-\xi),\right.
$$

so that

Copyright (C) by SIAM. Unauthorized reproduction of this article is prohibited. 


$$
\begin{aligned}
\left\{|\xi|^{2} a, i p\right\}= & 2 a|\xi|\left(\frac{\psi_{0}}{\langle x\rangle}\left(1-\operatorname{sign}(\xi) \frac{q x}{\langle x\rangle^{2}}\right)+\psi_{+} /\langle x\rangle^{1+\varepsilon}+\psi_{-} /\langle x\rangle^{1+\varepsilon}\right) \\
& +2 a|\xi|\left(q \psi_{0}^{\prime} /\langle x\rangle^{4}+(2 \delta+f(|q|)) \cdot\left(\psi_{+}^{\prime}+\psi_{+}^{\prime}(-x,-\xi)\right) /\langle x\rangle^{3}\right) .
\end{aligned}
$$

If $\psi_{0}(q /\langle x\rangle) \neq 0$ we have $|q| /\langle x\rangle \leq \delta$; therefore $\psi_{0} /\langle x\rangle\left(1-\operatorname{sign}(\xi) \frac{q x}{\langle x\rangle^{2}}\right) \geq$ $\psi_{0} /(2\langle x\rangle)$ for $\delta$ small enough.

Using that $\psi_{0}^{\prime}=\psi_{+}^{\prime}-\psi_{+}^{\prime}(-x,-\xi)$ and the support condition on $\psi_{0}$ we obtain

$$
q \psi_{0}^{\prime} /\langle x\rangle^{4} \geq \delta /\langle x\rangle^{3}\left(-\psi_{+}^{\prime}-\psi_{+}^{\prime}(-x,-\xi)\right),
$$

which implies

$$
2 a|\xi|\left(q \psi_{0}^{\prime} /\langle x\rangle^{4}+(2 \delta+f(|q|)) \cdot\left(\psi_{+}^{\prime}+\psi_{+}^{\prime}(-x,-\xi)\right) /\langle x\rangle^{3}\right) \geq 0 .
$$

We finally deduce the inequality

$$
\left\{|\xi|^{2} a, i p\right\} \geq 2 a|\xi|\left(\frac{\psi_{0}}{2\langle x\rangle}+\frac{\psi_{+}}{\langle x\rangle^{1+\varepsilon}}+\frac{\psi_{-}}{\langle x\rangle^{1+\varepsilon}}\right) \geq \frac{a|\xi|}{\langle x\rangle^{1+\varepsilon}} .
$$

Appendix B. A weighted Gårding inequality for nonsmooth symbols. This result is a direct adaptation of a version for an operator of $\Gamma_{\rho}^{1 / 2}$ in [ABZ].

Proposition B.1. Let $T_{c}$ be a paradifferential operator such that

$$
c(x, \xi) \geq K|\xi| /\langle x\rangle^{1+\delta}, c \in \Gamma_{1}^{1}
$$

then

$$
\exists K_{1}, K_{2}>0:\left\langle T_{c} u, u\right\rangle \geq K_{1}\left\|u /\langle x\rangle^{1+\delta}\right\|_{H^{1 / 2}}^{2}-K_{2}\|u\|_{L^{2}}^{2} .
$$

Proof. Since the symbol $c$ is not in $\Gamma_{2}^{1}$ we cannot directly apply the usual sharp Gårding's inequality. The method consists rather in reducing the analysis to a simple elliptic estimate as for the Gårding's inequality. It suffices to check that

$$
\left\langle T_{c} u, u\right\rangle \geq c \sum 1 / 2^{j(1+\delta)}\left\|\theta_{j} u\right\|_{H^{1 / 2}}^{2}-C\|u\|_{L^{2}}^{2},
$$

where $\left(\theta_{j}\right)$ is a sequence of functions $C_{c}^{\infty}\left(\mathbb{R} ; \mathbb{R}^{+}\right)$such that

$$
\sum_{j=0}^{\infty} \theta_{j}^{2}=1, \operatorname{supp}\left(\theta_{0}\right) \subset B(0,2), \theta_{j}=\theta\left(2^{-j} \cdot\right), \operatorname{supp}(\theta) \subset\{1 / 2 \leq|x| \leq 2\} .
$$

Let us write

$$
\left\langle T_{c} u, u\right\rangle=\sum_{0}^{\infty}\left\langle T_{c} \theta_{j}^{2} u, u\right\rangle
$$

Let $\Theta$ be a $C_{c}^{\infty}$ function such that $0 \notin \operatorname{supp}(\Theta), \Theta=1$ on $\operatorname{supp}(\theta)$. We also define $\Theta_{j}=\Theta\left(2^{-j}.\right)$, as well as $\Theta_{0} \in C_{c}^{\infty}$ with $\Theta_{0}=1$ on $\operatorname{supp}\left(\theta_{0}\right)$. Then 


$$
\begin{aligned}
\sum_{0}^{\infty}\left\langle T_{c} \theta_{j}^{2} u, u\right\rangle= & \sum_{0}^{\infty}\left\langle T_{c} \theta_{j}^{2} u, \Theta_{j} u\right\rangle+\left\langle T_{c} \theta_{j}^{2} u,\left(1-\Theta_{j}\right) u\right\rangle \\
= & \sum_{0}^{\infty}\left\langle T_{c} \theta_{j} u, \theta_{j} \Theta_{j} u\right\rangle+\left\langle\left[T_{c}, \theta_{j}\right] \theta_{j} u, \Theta_{j} u\right\rangle \\
& +\left\langle\theta_{j} u, \theta_{j} T_{c}^{*}\left(1-\Theta_{j}\right) u\right\rangle \\
= & \sum_{0}^{\infty}\left\langle\Theta_{j} T_{c} \theta_{j} u, \theta_{j} u\right\rangle+I_{1}+I_{2} \\
= & \sum_{0}^{\infty}\left\langle T_{c \Theta_{j}} \theta_{j} u, \theta_{j} u\right\rangle+I_{1}+I_{2}+\left\langle\left(\Theta_{j} T_{c}-T_{c \Theta_{j}}\right) \theta_{j} u, \theta_{j} u\right\rangle \\
= & \sum_{0}^{\infty}\left\langle T_{c \Theta_{j}} \theta_{j} u, \theta_{j} u\right\rangle+I_{1}+I_{2}+I_{3} .
\end{aligned}
$$

By hypothesis we have $c \Theta_{j} \geq 0$; in particular its square root $r:=\sqrt{c \Theta_{j}}$ is real positive. The rules of paradifferential calculus then imply

$$
T_{r}\left(T_{r}^{*}\right)=T_{c \Theta_{j}}+R_{j},
$$

where $R_{j}$ is a continuous operator $L^{2} \rightarrow H^{1 / 2+1 / 2-1}=L^{2}$, with a constant of continuity bounded uniformly in $j$. This gives

$$
\sum_{0}^{\infty}\left\langle T_{c \Theta_{j}} \theta_{j} u, \theta_{j} u\right\rangle=\sum_{0}^{\infty}\left\|T_{r} \theta_{j} u\right\|_{L^{2}}^{2}+\left\langle R_{j} \theta_{j} u, \theta_{j} u\right\rangle .
$$

The symbol $r$ satisfies $r \geq 2^{-j(1+\delta) / 2}|\xi|^{1 / 2} \Theta_{j}$ (for $\sqrt{\cdot} \geq I_{d}$ on $[0,1]$ ). In particular $r+\left(1-\Theta_{j}\right) 2^{-j(1+\delta) / 2}|\xi|^{1 / 2}$ is elliptic of order $1 / 2$. We deduce that

$$
\left\|T_{r} \theta_{j} u\right\|_{L^{2}}^{2} \geq c 2^{-j(1+\delta)}\left\|\theta_{j} u\right\|_{H^{1 / 2}}^{2}-C\left\|T_{\left(1-\Theta_{j}\right) 2^{-j(1+\delta) / 2}|\xi|^{1 / 2}} \theta_{j} u\right\|_{L^{2}}^{2},
$$

which is (B.1) up to neglectable terms. There remains to quantify what is meant by "neglectable."

$$
I_{1}=\sum_{0}^{\infty}\left\langle\left[T_{c}, \theta_{j}\right] \theta_{j} u, \Theta_{j} u\right\rangle \lesssim \sum_{0}^{\infty}\left\|\theta_{j} u\right\|_{L^{2}}^{2}+\left\|\Theta_{j} u\right\|_{L^{2}}^{2} \lesssim\|u\|_{L^{2}}^{2} .
$$

Since $\Theta_{j}$ is a real smooth bounded function as well as all its derivatives uniformly in $j$, the operator $T_{\Theta_{j}}-\Theta_{j}$ is continuous $L^{2} \rightarrow H^{1}$. Consequently $\Theta_{j} T_{c}-T_{c \Theta_{j}}$ is continuous $L^{2} \rightarrow L^{2}$ and we have

$$
I_{3}=\sum\left\langle\left(\Theta_{j} T_{c}-T_{c \Theta_{j}}\right) \theta_{j} u, \theta_{j} u\right\rangle \lesssim \sum\left\|\theta_{j} u\right\|_{L^{2}}^{2} \lesssim\|u\|_{L^{2}}^{2} .
$$

For the control of $I_{2}=\left\langle\theta_{j} u, \theta_{j} T_{c}^{*}\left(1-\Theta_{j}\right) u\right\rangle$ we must use a property of "paralocalization": denote $d \in \Gamma_{\rho}^{1}$ the symbol of $T_{c}^{*}$,

$$
\begin{aligned}
& \theta_{j} T_{d}\left(1-\Theta_{j}\right) u= \frac{1}{2 \pi} \iint e^{i(x \xi-y \eta)} \theta_{j}(x)\left(1-\Theta_{j}(y)\right) \\
& \widehat{d}(\xi-\eta, \eta) \psi(\eta) \chi(\xi-\eta, \eta) u(y) d y d \eta d \xi \frac{1}{2 \pi} \iint e^{i(x-y) \eta+i x \zeta} \theta_{j}(x)\left(1-\Theta_{j}(y)\right) \\
& \widehat{d}(\zeta, \eta) \psi(\eta) \chi(\zeta, \eta) u(y) d y d \eta d \xi .
\end{aligned}
$$

Copyright $\odot$ by SIAM. Unauthorized reproduction of this article is prohibited. 
First note that by definition of $\theta_{j}, \Theta_{j}$, on the support of the integrand $|x-y| \gtrsim 2^{j}$. An integration by parts on the variable $\eta$ gives

$$
\begin{array}{r}
\theta_{j} T_{c}^{*}\left(1-\Theta_{j}\right) u=-\frac{1}{2 \pi} \iint \frac{e^{i(x-y) \eta+i x \zeta}}{i(x-y)} \theta_{j}(x)\left(1-\Theta_{j}(y)\right) \\
\partial_{\eta}(\widehat{d}(\zeta, \eta) \psi(\eta) \chi(\zeta, \eta)) u(y) d y d \eta d \zeta \\
=-\frac{1}{2 \pi} \iint \frac{e^{i(x-y) \eta+i x \zeta} u(y)}{i(x-y)} \theta_{j}(x)\left(1-\Theta_{j}(y)\right) \\
\partial_{\eta}(\widehat{d}(\zeta, \eta) \psi(\eta) \chi(\zeta, \eta)) u(y) d y d \eta d \zeta
\end{array}
$$

We have

$$
\begin{aligned}
\partial_{\eta}(\widehat{d}(\zeta, \eta) \psi(\eta) \chi(\zeta, \eta))= & \psi^{\prime}(\eta) \widehat{d}(\zeta, \eta) \chi(\zeta, \eta)+\partial_{\eta} \widehat{d}(\zeta, \eta) \psi(\eta) \chi(\zeta, \eta) \\
& +\widehat{d}(\zeta, \eta) \psi(\eta) \partial_{\eta} \chi(\zeta, \eta) .
\end{aligned}
$$

The first term has compact support in $\eta$ because $\psi=1$ for $|x| \geq 2$ and consequently its derivative is fast decaying in $\eta$. The second term is of order 0 in $\eta$ because $d$ is of order 1 , and the third term is of order 0 in $\eta$, because (by homogeneity) $\partial_{\eta} \chi$ is of order -1 .

We also note that $\theta_{j}(x)\left(1-\Theta_{j}(y)\right) /|x-y| \lesssim 2^{-j}$; thus $\theta_{j} T_{c}^{*}\left(1-\Theta_{j}\right) u$ is in $L^{2}$, its norm being moreover controlled by $2^{-j}\|u\|_{L^{2}}$.

Finally we obtain

$$
\sum_{0}^{\infty}\left\langle\theta_{j} u, \theta_{j} T_{c}^{*}\left(1-\Theta_{j}\right) u\right\rangle \lesssim \sum_{0}^{\infty} 2^{-j}\|u\|_{L^{2}}^{2}+\left\|\theta_{j} u\right\|_{L^{2}}^{2} \lesssim\|u\|_{L^{2}}^{2} .
$$

The last term $\left\|T_{\left(1-\Theta_{j}\right) 2^{-j(1+\delta) / 2}|\xi|^{1 / 2}} \theta_{j} u\right\|_{L^{2}}^{2}$ can be treated in the same way as $I_{2}$, or more simply by writing

$$
\begin{aligned}
T_{\left(1-\Theta_{j}\right) 2^{-j(1+\delta) / 2}|\xi|^{1 / 2} \theta_{j} u} & =T_{\left(1-\Theta_{j}\right) 2^{-j(1+\delta) / 2}|\xi|^{1 / 2}} \theta_{j} \Theta_{j} u \\
& =T_{\theta_{j}\left(1-\Theta_{j}\right) 2^{-j(1+\delta) / 2}|\xi|^{1 / 2} \Theta_{j} u+R_{j} \Theta_{j} u} \\
& =R_{j} \Theta_{j} u
\end{aligned}
$$

with $R_{j}$ bounded from $L^{2}$ to $L^{2}$.

Appendix C. Construction of $\boldsymbol{p}$ (general case). This section is devoted to the construction of the symbol $p$ such that

$$
\left\{a|\xi|^{2}, p\right\} \geq c \frac{|\xi|}{\langle x\rangle^{1+\varepsilon}}-C .
$$

The scheme of construction is to construct a function $q(x, \xi)$ such that $|q| \lesssim\langle x\rangle$ and $\left\{a_{0}|\xi|^{2}, p\right\} \geq|\xi|-C$. This implies the existence of $p$ such that $\left\{a_{0}|\xi|^{2}, p\right\} \geq c \frac{|\xi|}{\langle x\rangle^{1+\varepsilon}}-C$, and we finally check that $p$ satisfies (C.1) for $t$ small enough.

We recall our assumptions:

- $a(x, t) \in C_{t} H^{s+1} \cap C_{t}^{1} H^{s}$, and $\left|\partial_{t} a\right|+\left|\partial_{t} \nabla a\right|+|\nabla a| \lesssim 1 /\langle x\rangle^{1+\varepsilon}$.

- The Hamiltonian $a_{0}(x)|\xi|^{2}:=a\left(\zeta_{0}\right)|\xi|^{2}$ has no trapped bicharacteristics.

Copyright $(\odot$ by SIAM. Unauthorized reproduction of this article is prohibited. 
Remark C.1. If $(\underline{\rho}, \underline{u})$ is a particular soliton solution, the decay assumption is satisfied by $\underline{a}:=a(\rho)$ only in the direction of propagation. In particular for traveling waves only depending of $x \cdot n-c t, a$ is constant on any affine hyperplane orthogonal to $n$, and the construction of this section does not apply. We denote $a_{0}(x)=a\left(\zeta_{0}(x)\right)$ with $\zeta_{0}$ the initial data. As a first step, we prove that there exists $q$ such that

$$
\left\{\begin{array}{l}
\left\{a_{0}|\xi|^{2}, q\right\} \geq c|\xi|-C, \\
\forall \alpha, \beta,\left|\partial_{x}^{\alpha} \partial_{\xi}^{\beta} q\right| \lesssim\langle x\rangle\langle\xi\rangle^{-|\beta|} .
\end{array}\right.
$$

Let $q_{1}=x \cdot \xi /|\xi|$; the decay assumption $\nabla a_{0} \leq C /\langle x\rangle^{1+\varepsilon}$ gives

$$
\begin{aligned}
\left\{a_{0}|\xi|^{2}, x \cdot \xi /|\xi|\right\} & =\sum_{j=1}^{d} 2 a_{0} \xi_{j} \xi_{j} /|\xi|-\partial_{x_{j}} a_{0}|\xi|^{2}\left(x_{j} /|\xi|-x \cdot \xi \xi_{j} /|\xi|^{3}\right) \\
& =2 a_{0}|\xi|-|\xi| \nabla a_{0} \cdot x+x \cdot \xi \nabla a_{0} \cdot \xi /|\xi| \\
& \geq a_{0}|\xi| \text { for } x \text { large enough. }
\end{aligned}
$$

Say that this inequality is valid for $|x| \geq M$, and take $\psi \in C^{\infty}\left(\mathbb{R}^{+}\right)$nondecreasing, equal to 0 on $[0, M]$ and 1 on $[M+1, \infty[$; then

$$
\begin{aligned}
\left\{a_{0}|\xi|^{2}, \psi\left(|x|^{2}\right) x \cdot \xi /|\xi|\right\} & =\psi\left(|x|^{2}\right)\left\{a_{0}|\xi|^{2}, x \cdot \xi /|\xi|\right\}+x \cdot \xi /|\xi|\left\{a_{0}|\xi|^{2}, \psi\left(|x|^{2}\right)\right\} \\
& \geq \psi\left(|x|^{2}\right) a_{0}|\xi|+2 a_{0}(x \cdot \xi)^{2} \psi^{\prime}\left(|x|^{2}\right) /|\xi| \\
& \geq \psi\left(|x|^{2}\right) a_{0}|\xi| .
\end{aligned}
$$

This construction works as well if we replace $a_{0}$ by $a$; it will not be the case for the second part.

By bilinearity of the Poisson bracket, it remains to find a symbol whose Poisson's bracket is positive for $x$ small. We recall that the bicharaceristics are the solutions of

$$
\left\{\begin{array}{l}
\dot{X}\left(t, x_{0}, \xi_{0}\right)=2 a_{0}(X) \Xi, t \geq 0, \\
\dot{\Xi}\left(t, x_{0}, \xi_{0}\right)=-|\Xi|^{2} \nabla a_{0}(X), t \geq 0, \\
X\left(0, x_{0}, \xi_{0}\right)=x_{0}, \Xi\left(0, x_{0}, \xi_{0}\right)=\xi_{0},
\end{array}\right.
$$

and assume that the bicharacteristics are not trapped, that is, for any $\left(x_{0}, \xi_{0}\right) \in$ $\mathbb{R}^{d} \times\left(\mathbb{R}^{d} \backslash\{0\}\right)$ we have $\left|X\left(t, x_{0}, \xi_{0}\right)\right| \rightarrow_{t \rightarrow+\infty}+\infty$.

Remark C.2. In dimension greater than 1, nontrapping is not an empty assumption. Even in the elementary case of a diagonal, asymptotically flat Laplacian, trapping may occur, as we can see, for instance, on the symbol

$$
a(x)|\xi|^{2}:=\left(\chi(x) e^{x_{1}^{2}+x_{2}^{2}}+\varphi\right)|\xi|^{2},
$$

where $\chi=1$ for $|x| \leq 5, \chi=0$ for $|x| \geq 6, \varphi=0$ for $|x| \leq 4$, and $\varphi=1$ for $|x| \geq 5$. It is easy to check that $X:=(\cos (2 e t), \sin (2 e t)), \Xi:=(-\sin (2 e t), \cos (2 e t))$ is a bounded solution of

$$
\left\{\begin{array}{l}
\dot{X}\left(t, x_{0}, \xi_{0}\right)=2 a_{0}(X) \Xi, t \geq 0 \\
\dot{\Xi}\left(t, x_{0}, \xi_{0}\right)=-|\Xi|^{2} \nabla a(X), t \geq 0 \\
X\left(0, x_{0}, \xi_{0}\right)=\left(\begin{array}{l}
1 \\
0
\end{array}\right), \Xi\left(0, x_{0}, \xi_{0}\right)=\left(\begin{array}{l}
0 \\
1
\end{array}\right)
\end{array}\right.
$$

Let us define

$$
q_{2}(x, \xi):=-\psi_{1}(x) \psi_{2}(\xi) \int_{0}^{\infty} \psi_{1}(X(s, x, \xi))\langle\Xi(s, x, \xi)\rangle d s,
$$

Copyright $@$ by SIAM. Unauthorized reproduction of this article is prohibited. 
where $\psi_{1} \in C_{c}^{\infty}$ is equal to 1 for $|x| \leq M+1$ and $\psi_{2} \in C^{\infty}$ is equal to 1 for $|\xi| \geq 1$ and vanishes on a neighborhood of 0 . Because of the nontrapping assumption $q_{2}$ is well defined. Moreover, by homogeneity in $\xi$ of the symbol $a(x)|\xi|^{2}$, we have

$$
(X(s, x, \xi), \Xi(s, x, \xi))=(X(s|\xi|, x, \xi /|\xi|),|\xi| \Xi(s|\xi|, x, \xi /|\xi|)) .
$$

Thus, setting $s|\xi|=s^{\prime}$,

$$
q_{2}(x, \xi)=-\frac{\psi_{1}(x) \psi_{2}(\xi)}{|\xi|} \int_{0}^{\infty} \psi_{1}\left(X\left(s^{\prime}, x, \xi /|\xi|\right)\right)\left\langle|\xi| \Xi\left(s^{\prime}, x, \xi /|\xi|\right)\right\rangle d s
$$

defines a symbol ${ }^{2}$ of order 0 . Its Poisson bracket with $a|\xi|^{2}$ satisfies

$$
\begin{aligned}
\left\{a_{0}|\xi|^{2}, q_{2}\right\}= & -\sum_{j=1}^{d} a_{0} 2 \xi_{j}\left(\partial_{x_{j}} \psi_{1}(x)\right) \psi_{2} \int_{0}^{\infty} \psi_{1}(X(s, x, \xi))\langle\Xi(s, x, \xi)\rangle d s \\
& +\sum_{j=1}^{d}|\xi|^{2} \partial_{x_{j}} a_{0} \psi_{1} \partial_{\xi_{j}} \psi_{2} \int_{0}^{\infty} \psi_{1}(X(s, x, \xi))\langle\Xi(s, x, \xi)\rangle d s \\
& +2\langle\xi\rangle \psi_{1}^{2}(x) \psi_{2}(\xi) .
\end{aligned}
$$

Since $\partial_{x_{j}} \psi_{1}$ vanishes on $B(0, M+1)$ and $\partial_{\xi_{j}} \psi_{2}$ is compactly supported, we have

$$
\left\{a|\xi|^{2}, q_{2}\right\} \geq-C\left(|\xi| 1_{|x| \geq M+1}+1\right)+\langle\xi\rangle \psi_{1}^{2}(x) \psi_{2}(\xi) .
$$

Taking $M=(C+1) / \inf \left(a_{0}\right)$, we have

$$
\left\{a_{0}|\xi|^{2}, M q_{1}+q_{2}\right\} \geq|\xi| 1_{|x| \geq M+1}-C+\langle\xi\rangle \psi_{1}(x)^{2} \psi_{2}(\xi) \geq|\xi|-C^{\prime} .
$$

Then a standard argument (in fact, an elementary generalization of the proof in the one-dimensional case where $M q_{1}+q_{2}$ would play the role of $q$; see also the very clear lecture notes of Kenig [Ken05, pp. 48-49]) gives the existence of $p$ such that

$$
\left\{a_{0}|\xi|^{2}, p\right\} \geq c \frac{|\xi|}{\langle x\rangle^{1+\varepsilon}}-C .
$$

Now assume also that $\left|\partial_{t} a\right|+\left|\partial_{t} \nabla a\right| \lesssim 1 /\langle x\rangle^{1+\varepsilon}$. This implies

$$
\left\{a|\xi|^{2}, p\right\} \geq c \frac{|\xi|}{\langle x\rangle^{1+\varepsilon}}-C+\left\{\int_{0}^{t} \partial_{t} a|\xi|^{2}, p\right\} \geq c \frac{|\xi|}{2\langle x\rangle^{1+\varepsilon}}-2 C
$$

for $t$ small enough.

Acknowledgments. Most of this work was done as part of the author's thesis at the University Lyon 1 under the guidance of Sylvie Benzoni-Gavage.

The author thanks the reviewers for a large number of valuable comments and corrections.

\footnotetext{
${ }^{2}$ The smoothness of the symbol is a consequence of the uniform nontrapping, i.e. for $(x, \xi)$ in a neighborhood of $\left(x_{0}, \xi_{0}\right)$, the bicharacteristics $(X(s, x, \xi), \Xi(s, x, \xi))$ leave any compact $K$ for $s \geq s(K)$ independent of $(x, \xi)$. This is a nontrivial but standard consequence of the nontrapping assumption combined with the decay assumption for $\nabla a$.
}

Copyright @ by SIAM. Unauthorized reproduction of this article is prohibited. 


\section{REFERENCES}

[ABZ] T. Alazard, N. Burq, And C. Zuily, On the Cauchy problem for the water waves with surface tension, D̀uke Math. J., 158 (2011), pp. 413-499.

[BGDD06] S. Benzoni-Gavage, R. Danchin, and S. Descombes, Well-posedness of onedimensional Korteweg models, Electron. J. Differential Equations, 59 (2006), pp. 1-35.

[BGDD07] S. Benzoni-Gavage, R. Danchin, and S. Descombes, On the well-posedness for the Euler-Korteweg model in several space dimensions, Indiana Univ. Math. J., 56 (2007), pp. 1499-1579.

[BGDDj05] S. Benzoni-Gavage, R. Danchin, S. Descombes, and D. Jamet, Structure of Korteweg models and stability of diffuse interfaces, Interfaces Free Bound., 7 (2005), pp. 371-414.

[BGDDJ07] S. Benzoni-Gavage, R. Danchin, S. Descombes, and D. Jamet, Stability issues in the Euler Korteweg model, in Control Methods in PDE-Dynamical Systems, AMS, Providence, RI, 2007, pp. 103-127.

[Bon81] J.-M. Bony, Calcul symbolique et propagation des singularités pour les équations aux dérivées partielles non linéaires, Ann. Sci. École Norm. Sup. (4), 14 (1981), pp. 209-246.

[CKS95] W. Craig, T. Kappeler, and W. Strauss, Microlocal dispersive smoothing for the Schrödinger equation, Comm. Pure Appl. Math., 48 (1995), pp. 769-860.

[CS88] P. Constantin AND J.-C. SAUT, Local smoothing properties of dispersive equations, J. Amer. Math. Soc., 1 (1988), pp. 413-439.

[Doi96] S.-I. DoI, Remarks on the Cauchy problem for Schrödinger-type equations, Comm. Partial Differential Equations, 21 (1996), pp. 163-178.

[Kat83] T. Kato, On the Cauchy problem for the (generalized) Korteweg-de Vries equation, in Studies in Applied Mathematics, Adv. Math. Suppl. Stud., Academic Press, New York, 1983, pp. 93-128.

[Ken05] C. Kenig, The Cauchy Problem for the Quasi-linear Schrödinger Equation, Lecture notes for the Park City Math Institute, http://www.math.brown.edu/holmer/ lectures/kenigi.pdf (2005).

[KF83] S. N. KRUZhKov AND A. V. FAminskil, Generalized solutions of the Cauchy problem for the Korteweg-de Vries equation, Mat. Sb. (N.S.), 120 (1983), pp. 396-425.

[KPV91] C. E. Kenig, G. Ponce, And L. Vega, Well-posedness of the initial value problem for the Korteweg-de Vries equation, J. Amer. Math. Soc., 4 (1991), pp. 323-347.

[KPV04] C. E. Kenig, G. Ponce, And L. Vega, The Cauchy problem for quasi-linear Schrödinger equations, Invent. Math., 158 (2004), pp. 343-388.

[Mét08] G. MÉtivier, Para-Differential Calculus and Applications to the Cauchy Problem for Nonlinear Systems, Centro di Ricerca Matematica Ennio De Giorgi Series 5. Edizioni della Normale, Pisa, 2008.

[Miz81] S. Mizohata, On some Schrödinger type equations, Proc. Japan Acad. Ser. A Math. Sci., 57 (1981), pp. 81-84.

Copyright $@$ by SIAM. Unauthorized reproduction of this article is prohibited. 